\title{
Quantum dynamical entropies for discrete classical systems: a comparison
}

\author{
VALERIO CAPPELLINI \\ Dipartimento di Fisica Teorica \\ Università di Trieste \\ Strada Costiera 11, 34014 Trieste, Italy \\ valerio.cappellini@ts.infn.it
}

1st October 2018

\begin{abstract}
On a family of classical dynamical systems on the 2-torus, we perform a discretization procedure similar to the Anti-Wick quantization. Such a discretization is performed by using a particular class of states, fulfilling an appropriate dynamical localization property, typical of quantum Coherent States. The same set of states is involved in the construction of a quantum entropy, that we test on the discrete approximants; a correspondence with the classical metric entropy of Kolmogorov-Sinai is found only over time scales that are logarithmic in the discretization parameter.
\end{abstract}

Short Title: Quantum dynamical entropies for discrete classical systems

Keywords: KS-Entropy, Quantum Dynamical Entropies, Chaos, Discrete Systems

PACS numbers: 05.45.Ac, 05.45.Mt, 03.65.Fd, 45.05. $+\mathrm{x}$

Mathematics Subject Classification 2000: 37D20; 54C70, 28D20, 81Q20, 81R30

\section{Contents}

1 Introduction $\quad 2$

2 Classical Dynamical Systems and Phase-Space discretization 4

2.1 Discretization of phase-space . . . . . . . . . . . . . . . 6

2.2 Lattice States on $\mathcal{H}_{\mathcal{N}} \ldots \ldots \ldots \ldots \ldots \ldots \ldots$

2.3 Anti-Wick Discretization and its continuous limit on $\mathrm{T}^{2} \ldots \ldots$

3 Discretization of the Dynamics $\quad 9$

3.1 General properties of matrix actions on the plane . . . . . . . . . . . 9 
3.2 Algebraic description of discretized UMG . . . . . . . . . . . 13

4 Continuous limit of the dynamics $\quad 14$

4.1 Continuous limit of discretized UMG . . . . . . . . . . . . . . 15

5 Dynamical Entropy on Discrete Systems 22

5.1 A classical one: Kolmogorov-Sinai metric entropy . . . . . . . . . . . . . . 23

5.2 Dynamics and Information in the Quantum Setting . . . . . . . . . . . 23

5.3 CS Quantum Entropies . . . . . . . . . . . . . . . . . . 25

5.4 CS Entropies for discrete classical systems . . . . . . . . . . . . . 27

6 Conclusions $\quad 31$

A Sketch of the proofs of Propositions 3.1, 3.2 and 3.3 32

$\begin{array}{ll}\text { References } & 34\end{array}$

\section{Introduction}

Under the term of classical chaos goes a rich phenomenology of classical dynamical systems on a compact phase space characterized by a high sensitivity to initial conditions: if very small initial errors exponentially amplify during the temporal evolution, the systems is called chaotic [1-7]. Nevertheless, being the motion confined within a bounded region, the exponential divergence of trajectories has to be tested in a finite domain. This leads to define the (maximal) coefficient of such exponential amplification, which is called Lyapunov exponent, as $\xi:=\lim _{n \rightarrow \infty}(1 / n) \lim _{\delta \rightarrow 0} \log \left(\delta_{n} / \delta\right)$, where we consider the initial error $\delta$ growing as $\delta_{n}$ under a discrete-time evolution. When the amplification of errors is exponential, the Lyapunov exponent $\xi$ is positive and the system is classified as chaotic. $\xi=0$ is typical of regular time-evolutions, but this also happens if we forbid $\delta$ to go to zero; indeed, $\delta_{n} \leqslant \Delta$ and $\lim \frac{1}{n}$ vanishes. This occurs for instance in the case of quantum dynamical systems, where the uncertainly principle naturally endows the phase-space with a $\hbar$-dependent granularity, and the $\delta \rightarrow 0$ limit can not be achieved for finite $\hbar>0$, but only if we perform the classical limit $\hbar \rightarrow 0$ before the time one. Although this shows the non commutativity of the classical and the time limits $[2,6]$, the temporal evolution of a finite dimensional quantization compared with its classical counterpart exhibits a good agreement on a time-scale bounded by the so called breaking time $\tau_{\mathrm{B}}(\hbar)$ : usually, 
when the classical system is chaotic, $\tau_{\mathrm{B}}$ scales logarithmically in $\hbar[1,2,6,8-10]$, whereas for regular systems the scaling is $\hbar^{-\alpha}$ for some $\alpha>0[1]$.

A similar phenomena can be observed in discrete classical systems, that are obtained for instance by forcing a classical system to live on a square lattice of $N^{2}$ points, whose minimal spacing $a=\frac{1}{N}$ acts as a lower bound for $\delta \rightarrow 0$ : in this case $\frac{1}{N}$ plays in the discrete domain the same role that $\hbar$ plays in the quantum one and can be interpreted as a quantization-like parameter.

By using this analogy of behaviours between quantum and discrete classical systems, the study of the latters result quite interesting and promising, indeed we can get all benefits arising from classicality, that is the simplicity due to commutativity, and deeply inquire the chaotic property in this kinds of "toy models".

Since finite dimensional quantizations of classical dynamical systems have an algebraic formulation, this can be easily extended to discretization procedures when we restrict from the full matrix algebra of bounded operators on a Hilbert space, typical of quantum systems, to a commutative algebra of diagonal operators describing a classical system [11].

A very useful tool of the semi-classical analysis of quantum systems is represented by the use of Coherent States and a standard quantization scheme, the Anti-Wick one [12], is based on them: by mimicking this procedure we set up a discretization involving a class of states that we will refer to as Lattice States, suitably defined on our Hilbert space. Of course, in order to have a good quantization, the classical limit $\hbar \rightarrow 0$ has to be tested [13] and large part of this work has been devoted to give and prove a consistent definition of a continuous limit $N \rightarrow \infty$, suited for a reasonable algebraic discretization scheme.

A first result in this direction is that the convergence of the discrete to the continuous dynamics is due to a very special property of Lattice States, that is known as dynamical localization property [14].

We apply our discretization procedure to a well known class of classical systems [7], that are represented by integer-matrix action on the 2-torus; such systems can be rigorously divided into three families, namely hyperbolic, parabolic and elliptic, characterized by different chaotic properties. As expected, differences in the behaviour of the breakingtimes $\tau_{\mathrm{B}}(N)$ (now of discrete/continuous correspondence) are found on the three different regimes.

The Lyapunov exponent is zero on systems with finite number of states (both discrete and quantum) because it is an asymptotic quantity: an alternative approach is to 
inquire the chaotic properties of a system during its temporal evolution, and whether the system exhibits some kind of finite-time chaos. For classical dynamical systems the Pesin-Ruelle Theorem [15] establish a bridge between chaos and information, giving a relation between the Kolmogorov-Sinai metric entropy and the sum of all positive Lyapunov exponent. Moreover, although the metric entropy is defined as a (partial) entropy production on the long run $[7,16]$, such a partial entropy can be observed and analyzed even during the temporal evolution, that is on finite times.

With the aim of using entropy to detect chaos, several quantum dynamical entropies have been introduced. In a recent work [14], two of them, called CNT (Connes, Narnhofer and Thirring) [17] and ALF (Alicky, Lindblad and Fannes) [18] are showed to converge to the KS invariant (but only in a joint time and classical limit) when applied to the AntiWick quantization of the hyperbolic family of the classical dynamical systems mentioned above. Only the hypothesis of dynamical localization for Coherent States was used in obtaining that result. Instead of extending such a result to our discretization scheme, we directly study another quantum dynamical entropy, constructed by means of Coherent States and so called CS-quantum entropy [19].

What we show is that the CS-entropy production of a discrete classical system does converge to the KS-entropy production of the continuous limit, but only over time scales logarithmic in the quantization-like parameter $\frac{1}{N}$. This confirms the numerical results obtained in [20] for the ALF-entropy on a similar class of discrete systems, but within the Weyl quantization-like scheme instead of the Anti-Wick.

Finally, we divided the CS-quantum entropy in its dynamical and measure-dependent parts, and we show how the latter does not play a role in the (positive) entropy rate.

\section{Classical Dynamical Systems and Phase-Space discretiza- tion}

The typical description of a Classical Dynamical System is given by means of a measure space $\mathcal{X}$, the phase-space, endowed with the Borel $\sigma$-algebra of its measurable subsets and a normalized measure $\mu,(\mu(\mathcal{X})=1)$. The probability that phase-points belong to measurable subsets $E \subseteq \mathcal{X}$ is given by the "volumes" $\mu(E)=\int_{E} \mu(\mathrm{d} \boldsymbol{x})$; so the measure $\mu$ defines the statistical properties of the system and represents a possible "state".

Every reversible discrete time dynamics amounts to an invertible measurable map $T: \mathcal{X} \mapsto \mathcal{X}$ such that $\mu \circ T=\mu$, and to its iterates $\left\{T^{k} \mid k \in \mathbb{Z}\right\}: T$-invariance of the measure $\mu$ ensure that the state defined by $\mu$ can be taken as an equilibrium state with 
respect to the given dynamics.

All phase-trajectories passing through $\boldsymbol{x} \in \mathcal{X}$ at time 0 can be encoded into sequences $\left\{T^{k} \boldsymbol{x}\right\}_{k \in \mathbb{Z}}[7]$.

Classical dynamical systems are thus conveniently described by measure-theoretic triplets $(\mathcal{X}, \mu, T)$. In particular, in the present work, we shall focus upon the following choices:

$\mathcal{X}$ : the 2-dimensional torus $\mathbb{T}^{2}=\mathbb{R}^{2} / \mathbb{Z}^{2}=\left\{\boldsymbol{x}=\left(x_{1}, x_{2}\right) \in \mathbb{R}^{2}(\bmod 1)\right\} ;$

$\mu$ : the Lebesgue measure, $\mu(\mathrm{d} \boldsymbol{x})=\mathrm{d} x_{1} \mathrm{~d} x_{2}$, on $\mathbb{T}^{2}$;

$T$ : the invertible measurable transformations on $\mathrm{T}^{2}$ represented by a modular matrix action, as follows:

$$
\begin{aligned}
T(\boldsymbol{x}) & =\left(\begin{array}{rr}
t_{11} & t_{12} \\
t_{21} & t_{22}
\end{array}\right)\left(\begin{array}{l}
x_{1} \\
x_{2}
\end{array}\right) \quad(\bmod 1), \quad \begin{array}{l}
t_{\imath \jmath} \in \mathbb{Z} \quad, \quad \forall(\imath, \jmath) \in\{1,2\}^{2} \\
\operatorname{det}(T)=t_{11} t_{22}-t_{21} t_{12}=1
\end{array} \\
T^{-1}(\boldsymbol{x}) & =\left(\begin{array}{rr}
t_{22} & -t_{12} \\
-t_{21} & t_{11}
\end{array}\right)\left(\begin{array}{l}
x_{1} \\
x_{2}
\end{array}\right) \quad(\bmod 1) \cdot
\end{aligned}
$$

\section{Remarks 2.1}

i. In the following, a point $\boldsymbol{x}$ of the torus, will correspond to an equivalence class of $\mathbb{R}^{2}$ points whose coordinates differ by integer values;

ii. in (1) we use brackets to distinguish between the mere matrix action $T \cdot \boldsymbol{x}$ and the $(\bmod 1)$ one $T(\boldsymbol{x})$;

iii. $T=\left(\begin{array}{ll}2 & 1 \\ 1 & 1\end{array}\right)$ is known as Arnold Cat Map [7], and it is an element of $\mathrm{SL}_{2}(\mathbb{Z}) \subset$ $\mathrm{GL}_{2}(\mathbb{Z}) \subset \mathrm{M}_{2}(\mathbb{Z})$, where the latter is the subset of $2 \times 2$ matrices with integer entries, $\mathrm{GL}_{2}(\mathbb{Z})$ the subset of invertible matrices and $\mathrm{SL}_{2}(\mathbb{Z})$ the subset of matrices with determinant one;

iv. the dynamics generated by $T \in \mathrm{SL}_{2}(\mathbb{Z})$, that is the one we are focusing on, is called Unimodular Group [7] (UMG for short);

v. $\operatorname{since} \operatorname{det}(T)=1$, the Lebesgue measure $\mu$ is invariant for all $T^{n} \in \mathrm{SL}_{2}(\mathbb{Z})$, $n \in \mathbb{Z}$.

In order to develop an algebraic discretization procedure as in [21], it proves convenient to follow an algebraic approach and replace $\left(\mathbb{T}^{2}, \mu, T\right)$ with the algebraic triple $\left(L_{\mu}^{\infty}\left(\mathrm{T}^{2}\right), \omega_{\mu}, \Theta\right)$, where 
$L_{\mu}^{\infty}\left(\mathrm{T}^{2}\right)$ is the (Abelian) Von Neumann *-algebra of (equivalence classes of) essentially bounded functions on $\mathbb{T}^{2}[22,23]$, equipped with the so-called essential supremum norm $\|\cdot\|_{\infty}[24]$

$\omega_{\mu}$ is the state (expectation) on $L_{\mu}^{\infty}\left(\mathrm{T}^{2}\right)$, defined by the reference measure $\mu$ as

$$
\omega_{\mu}: L_{\mu}^{\infty}\left(\mathbb{T}^{2}\right) \ni f \longmapsto \omega_{\mu}(f):=\int_{\mathbb{T}^{2}} \mu(\mathrm{d} \boldsymbol{x}) f(x) \in \mathbb{R}^{+}
$$

$\Theta$ is the automorphism of $L_{\mu}^{\infty}\left(\mathbb{T}^{2}\right)$ defined by $\Theta^{j}(f):=f \circ T^{j}$, satisfying $\omega \circ \Theta^{j}=\omega$.

\subsection{Discretization of phase-space}

From an algebraic point of view, a discretization procedure resembles very much quantization. Given the classical algebraic triple $\left(L_{\mu}^{\infty}\left(\mathrm{T}^{2}\right), \omega_{\mu}, \Theta\right)$, the core of a quantizationdequantization procedure (specifically an $\mathcal{N}$-dimensional quantization) is twofold:

- finding a pair of ${ }^{*}$-morphisms, $\mathcal{J}_{\mathcal{N}, \infty}$ mapping $L_{\mu}^{\infty}\left(\mathrm{T}^{2}\right)$ into a finite dimensional algebra $\mathcal{M}_{\mathcal{N}}$ (in general a full $N \times N$ matrix algebra) and $\mathcal{J}_{\infty, \mathcal{N}}$ mapping backward $\mathcal{M}_{\mathcal{N}}$ into $L_{\mu}^{\infty}\left(\mathrm{T}^{2}\right)$

- providing an automorphism $\Theta_{\mathcal{N}}$, the quantum dynamics, acting on $\mathcal{M}_{\mathcal{N}}$ such that it approximates in a suitable sense the classical one, $\Theta$, on $L_{\mu}^{\infty}\left(\mathbb{T}^{2}\right)$ as follows

$$
\mathcal{J}_{\infty, \mathcal{N}} \circ \Theta_{\mathcal{N}}^{j} \circ \mathcal{J}_{\mathcal{N}, \infty} \underset{N \rightarrow \infty}{\longrightarrow} \Theta^{j}
$$

The latter requirement can be seen as a modification of the so called Egorov's property (see [25]).

A similar procedure, that we will call discretization, can be obtained if we replace the full matrix algebra $\mathcal{M}_{\mathcal{N}}$ with a finite abelian one, namely the algebra $\mathcal{D}_{\mathcal{N}}$ consisting of $N^{2} \times N^{2}$ diagonal matrices.

In order to give to elements of $\mathcal{D}_{\mathcal{N}}$ the meaning of discrete observables, we define a suitable Hilbert space: to do this, we consider a discretized version of $\left(\mathbb{T}^{2}, \mu, T\right)$ which arises by forcing the continuous classical system to live on a square lattice $L_{N} \subseteq \mathbb{T}^{2}$ of spacing $\frac{1}{N}$ :

$$
L_{N}:=\left\{\frac{\boldsymbol{p}}{N} \mid \boldsymbol{p} \in(\mathbb{Z} / N \mathbb{Z})^{2}\right\},
$$

where $(\mathbb{Z} / N \mathbb{Z})$ denotes the residual class $(\bmod N)$, that is $0 \leqslant p_{i} \leqslant N-1$. 
Now we take the $\mathcal{N}:=N^{2}$ points of $L_{N}$ as labels of the elements $\{\mid \ell\}_{\ell \in(\mathbb{Z} / N \mathbb{Z})^{2}}$ of an orthonormal basis (o.n.b.) of the $\mathcal{N}$ dimensional Hilbert space $\mathcal{H}_{\mathcal{N}}$, and we consider discrete algebraic triples $\left(\mathcal{D}_{\mathcal{N}}, \tau_{\mathcal{N}}, \Theta_{\mathcal{N}}\right)$, consisting of

$\mathcal{D}_{\mathcal{N}}:$ an $\mathcal{N} \times \mathcal{N}$ matrix algebra diagonal in the orthonormal basis introduced above;

$\tau_{\mathcal{N}}$ : the uniform state (expectation) on $\mathcal{D}_{\mathcal{N}}$ defined by

$$
\tau_{\mathcal{N}}: \mathcal{D}_{\mathcal{N}} \ni D \longmapsto \tau_{\mathcal{N}}(D):=\frac{1}{\mathcal{N}} \operatorname{Tr}(D) \in \mathbb{R}^{+}
$$

$\Theta_{\mathcal{N}}$ : an automorphism of $\mathcal{D}_{\mathcal{N}}$ suitably reproducing $\Theta$ when $N \longrightarrow \infty$ (see Section 3.2).

In particular, as the Anti-Wick quantization can be obtained by means of Coherent States [12], a similar Anti-Wick discretization of $\left(L_{\mu}^{\infty}\left(\mathbb{T}^{2}\right), \omega_{\mu}, \Theta\right)$ in $\left(\mathcal{D}_{\mathcal{N}}, \tau_{\mathcal{N}}, \Theta_{\mathcal{N}}\right)$ can be performed [21] once that we specified what we consider as "Coherent States" on $\mathcal{H}_{\mathcal{N}}$, and this is the purpose of next Section.

Intuitively, a discrete description of $\left(\mathbb{T}^{2}, \mu, T\right)$ becomes finer when we increase $N$, the number of points per linear dimension on the grid $L_{N}$ in (3): this corresponds to enlarging the dimension of the Hilbert space $\mathcal{H}_{\mathcal{N}}$ associate to the corresponding algebraic triple $\left(\mathcal{D}_{\mathcal{N}}, \tau_{\mathcal{N}}, \Theta_{\mathcal{N}}\right)$. In this sense, the lattice spacing $a:=\frac{1}{N}$ of the grid $L_{N}$ is a natural discretization parameter playing an analogous role to the quantization parameter $\hbar$.

\section{2. $\quad$ Lattice States on $\mathcal{H}_{\mathcal{N}}$}

In analogy with the the properties of quantum Coherent States, we shall look for analogous states on the torus, that we shall call Lattice States [21]. For the benefits of the reader, we list below the set of properties which make quantum Coherent States such a useful tool in semiclassical analysis.

\section{Properties 2.1 (of Quantum Coherent States)}

A family $\left\{\left|C_{\mathcal{N}}(\boldsymbol{x})\right\rangle \mid \boldsymbol{x} \in \mathbb{T}^{2}\right\} \in \mathcal{H}_{\mathcal{N}}$ of vectors, indexed by points $\boldsymbol{x} \in \mathbb{T}^{2}$, constitutes a set of Coherent States on the torus if it satisfies the following requirements:

1. Measurability: $\boldsymbol{x} \mapsto\left|C_{\mathcal{N}}(\boldsymbol{x})\right\rangle$ is measurable on $\mathrm{T}^{2}$;

2. Normalization: $\left\|C_{\mathcal{N}}(\boldsymbol{x})\right\|^{2}=1, \boldsymbol{x} \in \mathbb{T}^{2}$;

3. Completeness: $\mathcal{N} \int_{\mathbb{T}^{2}} \mu(\mathrm{d} \boldsymbol{x})\left|C_{\mathcal{N}}(\boldsymbol{x})\right\rangle\left\langle C_{\mathcal{N}}(\boldsymbol{x})\right|=\mathbb{1}$; 
4. Localization: given $\varepsilon>0$ and $d_{0}>0$, there exists $N_{0}\left(\varepsilon, d_{0}\right)$ such that for $N \geq N_{0}\left(\varepsilon, d_{0}\right)$ and $d_{\mathbb{T}^{2}}(\boldsymbol{x}, \boldsymbol{y}) \geq d_{0}$ one has $\mathcal{N}\left|\left\langle C_{\mathcal{N}}(\boldsymbol{x}), C_{\mathcal{N}}(\boldsymbol{y})\right\rangle\right|^{2} \leq \varepsilon$.

The symbol $d_{\mathbb{T}^{2}}(\boldsymbol{x}, \boldsymbol{y})$ used in the localization property stands for the length of the shorter segment connecting the two points $\boldsymbol{x}, \boldsymbol{y} \in \mathbb{T}^{2}$, namely we shall denote by

$$
d_{\mathbb{T}^{2}}(\boldsymbol{x}, \boldsymbol{y}):=\min _{\boldsymbol{n} \in \mathbb{Z}^{2}}\|\boldsymbol{x}-\boldsymbol{y}+\boldsymbol{n}\|_{\mathbb{R}^{2}}
$$

the distance on $\mathbb{T}^{2}$.

\section{Remarks 2.2 (Topology of the UMG on the torus)}

i. Notice that $d_{\mathbb{T}^{2}}(\boldsymbol{a}, \boldsymbol{b})=\|\boldsymbol{a}-\boldsymbol{b}\|_{\mathbb{R}^{2}}$ if $\|\boldsymbol{a}-\boldsymbol{b}\|_{\mathbb{R}^{2}} \leqslant \frac{1}{2}$

ii. All the automorphisms $T \in \mathrm{SL}_{2}(\mathbb{Z})$ defined in (1) act continuously on the torus, when the topology is given by the distance (5).

Resorting to the decomposition $\mathbb{T}^{2} \ni \boldsymbol{x}=\left(\frac{\left\lfloor N x_{1}\right\rfloor}{N}, \frac{\left\lfloor N x_{2}\right\rfloor}{N}\right)+\left(\frac{\left\langle N x_{1}\right\rangle}{N}, \frac{\left\langle N x_{2}\right\rangle}{N}\right) \Rightarrow \frac{\lfloor N \boldsymbol{x}\rfloor}{N}+\frac{\langle N \boldsymbol{x}\rangle}{N}$, where $\lfloor\cdot\rfloor$ and $\langle\cdot\rangle$ denote the integer, respectively fractional, part of a real number, we now make use of the definition of the family $\left|C_{\mathcal{N}}(\boldsymbol{x})\right\rangle$ of Lattice States given in [21], that consists in associating to points of $\mathbb{T}^{2}$ specific lattice points (see [21], Fig. 1).

\section{Definition 2.1 (Lattice States)}

Given $\boldsymbol{x} \in \mathbb{T}^{2}$, we shall denote by $\hat{\boldsymbol{x}}_{N}$ the element of $(\mathbb{Z} / N \mathbb{Z})^{2}$ given by

$$
\hat{\boldsymbol{x}}_{N}=\left(\hat{x}_{N, 1}, \hat{x}_{N, 2}\right):=\left(\left\lfloor N x_{1}+\frac{1}{2}\right\rfloor,\left\lfloor N x_{2}+\frac{1}{2}\right\rfloor\right),
$$

and call Lattice States on $\mathbb{T}^{2}$ the vectors $\left|C_{\mathcal{N}}(\boldsymbol{x})\right\rangle$ defined by

$$
\mathbb{T}^{2} \ni \boldsymbol{x} \mapsto\left|C_{\mathcal{N}}(\boldsymbol{x})\right\rangle:=\left|\hat{\boldsymbol{x}}_{N}\right\rangle \in \mathcal{H}_{\mathcal{N}}
$$

The reader can check in [21] that family $\left\{\mid C_{\mathcal{N}}(\boldsymbol{x})\right\}$ satisfies Properties 2.1. In particular, in the last proof, it is also shown that, due to our particular choice of Lattice States, we have a stronger localization than in Property 2.1.4., namely

$4^{\prime}$. Localization: given $d_{0}>0$, there exists $N_{0}\left(d_{0}\right)$ such that for $N \geq N_{0}\left(d_{0}\right)$ and $d_{\mathbb{T}^{2}}(\boldsymbol{x}, \boldsymbol{y}) \geq d_{0}$ one has $\left\langle C_{\mathcal{N}}(\boldsymbol{x}), C_{\mathcal{N}}(\boldsymbol{y})\right\rangle=0$.

\subsection{Anti-Wick Discretization and its continuous limit on $\mathbb{T}^{2}$}

In order to study the continuous limit and, more generally, the quasi-continuous behaviour of $\left(\mathcal{D}_{\mathcal{N}}, \tau_{\mathcal{N}}, \Theta_{\mathcal{N}}\right)$ when $N \rightarrow \infty$, we follow the semi-classical technique known 
as Anti-Wick quantization. Therefore, we start choosing concrete discretization/dediscretization ${ }^{*}$-morphisms.

\section{Definitions 2.2}

Given the family of Lattice States $\left\{\left|C_{\mathcal{N}}(\boldsymbol{x})\right\rangle\right\} \in \mathcal{H}_{\mathcal{N}}$ of previous Section, the Anti-Wick-like discretization scheme (AW, for short) is described by a one parameter family of (completely) positive unital map $\mathcal{J}_{\mathcal{N}, \infty}: L_{\mu}^{\infty}\left(\mathbb{T}^{2}\right) \rightarrow \mathcal{D}_{\mathcal{N}}$

$$
L_{\mu}^{\infty}\left(\mathrm{T}^{2}\right) \ni f \mapsto \mathcal{N} \int_{\mathbb{T}^{2}} \mu(\mathrm{d} \boldsymbol{x}) f(\boldsymbol{x})\left|C_{\mathcal{N}}(\boldsymbol{x})\right\rangle\left\langle C_{\mathcal{N}}(\boldsymbol{x})\right|=: \mathcal{J}_{\mathcal{N}, \infty}(f) \in \mathcal{D}_{\mathcal{N}}
$$

The corresponding de-discretization operation is described by the (completely) positive unital map $\mathcal{J}_{\infty, \mathcal{N}}: \mathcal{D}_{\mathcal{N}} \rightarrow L_{\mu}^{\infty}\left(\mathbb{T}^{2}\right)$

$$
\mathcal{D}_{\mathcal{N}} \ni X \mapsto\left\langle C_{\mathcal{N}}(\boldsymbol{x}), X C_{\mathcal{N}}(\boldsymbol{x})\right\rangle=: \mathcal{J}_{\infty, \mathcal{N}}(X)(\boldsymbol{x}) \in L_{\mu}^{\infty}\left(\mathrm{T}^{2}\right)
$$

Both maps are identity preserving (unital) because of the conditions satisfied by the family of Lattice States and completely positive too, since both $L_{\mu}^{\infty}\left(\mathbb{T}^{2}\right)$ and $\mathcal{D}_{\mathcal{N}}$ are commutative algebras. The reader can found in [21] and [14] a list of simple properties of these maps, that incorporate minimal requests for rigorously defining the sense in which the discrete dynamical systems $\left(\mathcal{D}_{\mathcal{N}}, \tau_{\mathcal{N}}, \Theta_{\mathcal{N}}\right)$ tends to $\left(L_{\mu}^{\infty}\left(\mathbb{T}^{2}\right), \omega_{\mu}, \Theta\right)$, when $\frac{1}{N} \rightarrow 0$.

\section{Discretization of the Dynamics}

\subsection{General properties of matrix actions on the plane}

The next natural step in our discretization procedure will be the definition of a suitable discrete dynamics $\Theta_{\mathcal{N}}$ on the abelian algebra $\mathcal{D}_{\mathcal{N}}$ of Section 2.1. Before doing this we shall focus on some basic properties of the (integer) matrix action on the plane, that are

$$
\mathbb{R}^{2} \ni \boldsymbol{x} \longmapsto T \boldsymbol{x}=\left(\begin{array}{ll}
t_{11} & t_{12} \\
t_{21} & t_{22}
\end{array}\right)\left(\begin{array}{c}
x_{1} \\
x_{2}
\end{array}\right) \in \mathbb{R}^{2}, \quad \begin{aligned}
& t_{\imath \jmath} \in \mathbb{Z} \quad, \quad \forall(\imath, \jmath) \in\{1,2\}^{2} \\
& \operatorname{det}(T)=t_{11} t_{22}-t_{21} t_{12}=1
\end{aligned}
$$

Note that in this Section we begin by considering integer matrices $T$, with determinant one, mapping the plane onto itself; in Section 3.2 we will go back to actions on the torus $\mathbb{T}^{2}$, as in (1a).

\section{Definitions 3.1 (Families of matrix actions)}


We exclude from now on the cases $T= \pm \mathbb{1}_{2}$, the identity on the plane, that are trivial. Depending on the trace of $T$ we have three families of maps, characterized by their spectral properties; in particular, denoting with $t:=$ $\frac{\operatorname{Tr}(T)}{2}$ the semi-trace of $T$, the eigenvalues are given by $t \pm \sqrt{t^{2}-1}$ and we have:

$|\boldsymbol{t}|>\mathbf{1}$ - Hyperbolic family: One eigenvalue of $T, \lambda$, is greater than 1 (in modulus) and the other one is $\lambda^{-1}$. In this case, distances are stretched along the direction of the eigenvector $\left|\boldsymbol{e}_{+}\right\rangle, T\left|\boldsymbol{e}_{+}\right\rangle=\lambda\left|\boldsymbol{e}_{+}\right\rangle$, contracted along that of $\left|\boldsymbol{e}_{-}\right\rangle, T\left|\boldsymbol{e}_{-}\right\rangle=\lambda^{-1}\left|\boldsymbol{e}_{-}\right\rangle$. The (positive) Lyapunov exponent is given by $\xi=\log |\lambda|$.

$|\boldsymbol{t}|=\mathbf{1}$ - Parabolic family: There is only one eigenvalue, whose modulus is equal to one, which corresponds to an eigenvector $\left|\boldsymbol{e}_{0}\right\rangle$.

$|\boldsymbol{t}|<\mathbf{1}$ - Elliptic family: The two eigenvalues are conjugate complex numbers $e^{i \phi}$ and $e^{-i \phi}$, whose corresponding eigenvectors $\left|\boldsymbol{e}_{+}\right\rangle$and $\left|\boldsymbol{e}_{-}\right\rangle$are complex conjugate vectors of $\mathbb{C}^{2}$. On the (non-orthogonal) basis $\left\{\left|\boldsymbol{e}_{\mathrm{R}}\right\rangle,\left|\boldsymbol{e}_{\mathrm{I}}\right\rangle\right\}:=$ $\left\{\operatorname{Re}\left(\left|\boldsymbol{e}_{+}\right\rangle\right), \operatorname{Im}\left(\left|\boldsymbol{e}_{+}\right\rangle\right)\right\}, T^{n}$ is represented by means of the rotation matrix:

$$
R^{n}=\left(\begin{array}{rr}
\cos (n \phi) & \sin (n \phi) \\
-\sin (n \phi) & \cos (n \phi)
\end{array}\right)
$$

Before exploring the properties of the three regimes given above, we list now some more

\section{Definitions 3.2}

Let $B_{T}(0):=\left\{\boldsymbol{x} \in \mathbb{R}^{2} \mid\|\boldsymbol{x}\|_{\mathbb{R}^{2}} \leqslant 1\right\}$ be the unitary ball on the plane and

$$
B_{T}(p):=\left\{\boldsymbol{x} \in \mathbb{R}^{2} \mid T^{-p} \boldsymbol{x} \in B_{T}(0)\right\}
$$

be the $p$-evolved ball $(p \in \mathbb{Z})$. Then define as

$$
B_{T}^{(n)}:=\bigcup_{p=-n}^{n} B_{T}(p)
$$

the union of all evolved balls from time $-n$ up to time $n(n \in \mathbb{N})$ and let $D_{T}^{(n)}:=\operatorname{diam}\left[B_{T}^{(n)}\right]$ be its diameter, so as $D_{T}(p):=\operatorname{diam}\left[B_{T}(p)\right]$ will be the diameter of the $p$-evolved ball (diam $[E]:=\sup _{\boldsymbol{x}, \boldsymbol{y} \in E}\|\boldsymbol{x}-\boldsymbol{y}\|_{\mathbb{R}^{2}}$ ). Further, we denote by $\eta$ the largest eigenvalue of the matrix $|T|=\sqrt{T^{\dagger} T}$. 
Using this notation we now list three Propositions, one for each family, that incorporate the main properties; a sketch of their proofs is given in Appendix A. 


\section{Proposition 3.1 (Hyperbolic family)}

Let $T$ be a matrix belonging to the hyperbolic family of Definitions 3.1.

Without loss of generality we choose $\left|\boldsymbol{e}_{+}\right\rangle$and $\left|\boldsymbol{e}_{-}\right\rangle$of in such a way that the angle $\beta$ from the former to the latter lies in $(0, \pi)$ and we fix an orthogonal reference system $(\hat{\boldsymbol{x}}, \hat{\boldsymbol{y}})$ with $x$-axis oriented along the eigenvector $\left|\boldsymbol{e}_{+}\right\rangle$: in such a system all orbits of the (discrete) group $\left\{T^{k}\right\}_{k \in \mathbb{Z}}$ lie on hyperbolas

$$
y^{2} \cos \beta-x y \sin \beta=\text { Const. . }
$$

The angle $\beta$, whose sine is positive according to our choice of $\left|\boldsymbol{e}_{+}\right\rangle$and $\left|\boldsymbol{e}_{-}\right\rangle$, is related with $\eta$ of Definitions 3.2 by

$$
\sin \beta=\frac{\lambda-\lambda^{-1}}{\eta-\eta^{-1}}
$$

moreover, for every $n \in \mathbb{N}$, the set $B_{T}^{(n)}$ is confined into the hyperbolic region delimited by the four branches of the two hyperbolas

$$
2 y^{2} \cos \beta-2 x y \sin \beta-(\cos \beta \pm 1)=0 \quad .
$$

For the diameters, we have

$$
D_{T}^{(n)}=D_{T}(n)=\frac{\lambda^{n}-\lambda^{-n}}{2 \sin \beta}\left\{1+\sqrt{1+\left(\frac{2 \sin \beta}{\lambda^{n}-\lambda^{-n}}\right)^{2}}\right\}
$$

or, resorting to the expression for the Lyapunov exponent $\xi$ given in Definition 3.1:

$$
\sin \beta \sinh \left\{\log \left[D_{T}^{(n)}\right]\right\}=\sinh (n \xi)
$$

Moreover

$$
\forall n \in \mathbb{N} \quad, \quad D_{T}^{(n)} \leqslant \frac{\lambda^{n}}{\sin \beta} \quad \text { and } \quad D_{T}^{(n)} \underset{n \longrightarrow \infty}{\longrightarrow} \frac{\lambda^{n}}{\sin \beta} .
$$

\section{Proposition 3.2 (Parabolic family)}

Let $T$ be a matrix belonging to the parabolic family of Definitions 3.1.

We fix an orthogonal reference system $(\hat{\boldsymbol{x}}, \hat{\boldsymbol{y}})$ with $x$-axis oriented along the eigenvector $\left|\boldsymbol{e}_{0}\right\rangle$ : in such a system all orbits of the (discrete) group $\left\{T^{k}\right\}_{k \in \mathbb{Z}}$ 
lie on the

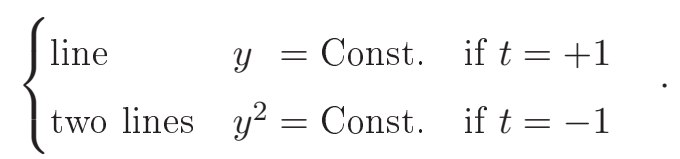

For every $n \in \mathbb{N}$ the set $B_{T}^{(n)}$ is confined into the stripe delimited by the two lines

$$
y^{2}=1
$$

Resorting to $\eta$ of Definitions 3.2, we introduce a positive real parameter

$$
J=\frac{\eta-\eta^{-1}}{2}
$$

that is used in the expression for the diameters, that is

$$
D_{T}^{(n)}=D_{T}(n)=n J+\sqrt{n^{2} J^{2}+1}
$$

or, equivalently,

$$
\sinh \left\{\log \left[D_{T}^{(n)}\right]\right\}=n J
$$

Moreover

$$
\forall n \in \mathbb{N} \quad, \quad D_{T}^{(n)} \leqslant 2 n J+1
$$

and

$$
D_{T}^{(n)} \underset{n \longrightarrow \infty}{\longrightarrow} 2 n J
$$

Proposition 3.3 (Elliptic family)

Let $T$ be a matrix belonging to the elliptic family of Definitions 3.1 ; if the entries of this matrix are integer, it holds true:

$$
\begin{array}{lrl}
\forall n \in \mathbb{N} & , \quad D_{T}(n) \leqslant \eta, \\
\forall n \in \mathbb{N}^{+}, & D_{T}^{(n)}=\eta,
\end{array}
$$

where $\eta$ is the one introduced in Definitions 3.2.

\subsection{Algebraic description of discretized UMG}

Our aim is now to define a suitable discrete evolution $\Theta_{\mathcal{N}}$ on $\mathcal{D}_{\mathcal{N}}$ (see Section 2.1 for the definitions), such that the discretized triplets $\left(\mathcal{D}_{\mathcal{N}}, \tau_{\mathcal{N}}, \Theta_{\mathcal{N}}\right)$ converge to the continuous 
one $\left(L_{\mu}^{\infty}\left(\mathbb{T}^{2}\right), \omega_{\mu}, \Theta\right)$.

We start by introducing a new family of maps $\left\{U_{T}^{j}\right\}_{j \in \mathbb{Z}}$, defined on the torus $\mathbb{T}^{2}([0, N))$, given by the action determined by the matrix $T(\bmod N)$, that is

$$
\mathbb{T}^{2}([0, N)) \ni \boldsymbol{x} \longmapsto U_{T}^{j}(\boldsymbol{x}):=N T^{j}\left(\frac{\boldsymbol{x}}{N}\right) \in \mathbb{T}^{2}([0, N)) \quad, \quad j \in \mathbb{Z},
$$

where $T(\cdot)$ is the map defined in (1). The $U_{T}^{j}(\cdot)$ maps are extensions of the $T^{j}(\cdot)$ maps on the enlarged torus $\mathbb{T}^{2}([0, N))$; moreover, they do map the lattice $(\mathbb{Z} / N \mathbb{Z})^{2}$ into itself, so as the maps $T^{j}(\cdot)$ do it with the lattice $L_{N}$ of $(3)$.

Note that the map $(\mathbb{Z} / N \mathbb{Z})^{2} \ni \boldsymbol{\ell} \longmapsto U_{T}(\ell) \in(\mathbb{Z} / N \mathbb{Z})^{2}$ is a bijection.

\section{Definition 3.3}

$\Theta_{\mathcal{N}}$ will denote the map:

$$
\mathcal{D}_{\mathcal{N}} \ni X \longmapsto \Theta_{\mathcal{N}}(X):=\sum_{\ell \in(\mathbb{Z} / N \mathbb{Z})^{2}} X_{U_{T}(\ell), U_{T}(\ell)}|\ell\rangle\langle\ell| \in \mathcal{D}_{\mathcal{N}}
$$

The map $\Theta_{\mathcal{N}}$ is a ${ }^{*}$-automorphism of $\mathcal{D}_{\mathcal{N}}$; indeed

$$
\begin{aligned}
\Theta_{\mathcal{N}}(X) & =\sum_{U_{T}^{-1}(\boldsymbol{s}) \in(\mathbb{Z} / N \mathbb{Z})^{2}} X_{\boldsymbol{s}, \boldsymbol{s}}\left|U_{T}^{-1}(\boldsymbol{s})\right\rangle\left\langle U_{T}^{-1}(\boldsymbol{s})\right|= \\
& =W_{T, N}\left(\sum_{\substack{\text { all equiv. } \\
\text { classes }}} X_{\boldsymbol{s}, \boldsymbol{s}}|\boldsymbol{s}\rangle\langle\boldsymbol{s}|\right) W_{T, N}^{*}= \\
& =W_{T, N} X W_{T, N}^{*},
\end{aligned}
$$

where the operators $W_{T, N}$, defined by linearly extending the maps

$$
\mathcal{H}_{\mathcal{N}} \ni|\ell\rangle \longmapsto W_{T, N}|\ell\rangle:=\left|U_{T}^{-1}(\ell)\right\rangle \in \mathcal{H}_{\mathcal{N}}
$$

to $\mathcal{H}_{\mathcal{N}}$, are unitary: $W_{T, N}^{*}|\ell\rangle:=\left|U_{T}(\ell)\right\rangle$. For the same reason $\tau_{\mathcal{N}}$ is a $\Theta_{\mathcal{N}}$-invariant state.

\section{Continuous limit of the dynamics}

One of the main issues in the semi-classical analysis is to compare if and how the quantum and classical time evolutions mimic each other when the quantization parameter goes to zero. 
In this paper we are instead considering the possible agreement between the dynamics of continuous classical systems and that of a class of discrete approximants. In practice, in our case, we will study the difference

$$
\Theta^{j}-\mathcal{J}_{\infty, \mathcal{N}} \circ \Theta_{\mathcal{N}}^{j} \circ \mathcal{J}_{\mathcal{N}, \infty}
$$

which represents how much the discrete dynamics at timestep $j$ differs from the continuous one at the same timestep.

For quantum systems, whose classical limit is chaotic, the situation is strikingly different from those with regular classical limit. In the former case, classical and quantum mechanics agree, that is a difference as in (28) is negligible, only over times $j$ which scale logarithmically (and not as a power law) in the quantization parameter.

As we shall see, such kind of scaling is not exclusively related with non-commutativity; in fact, the quantization-like procedure developed so far, exhibits a similar behaviour when $N \rightarrow \infty$ and we recover $\left(L_{\mu}^{\infty}\left(\mathbb{T}^{2}\right), \omega_{\mu}, \Theta\right)$ as a continuous limit of $\left(\mathcal{D}_{\mathcal{N}}, \tau_{\mathcal{N}}, \Theta_{\mathcal{N}}\right)$.

\subsection{Continuous limit of discretized UMG}

We want to show that the difference in (28) goes to zero in a suitable topology, at least on a certain time-scale. Such scales, commonly called breaking times, depend on the family of the considered map $T$. In the following, we give three different scaling functions of $n$, one per each family of matrix action, that will be compared with $\log N$ in the joint limits in $n$ and $N$ that we will construct in this Section.

\section{Definition 4.1}

We shall denote by $\Gamma_{T}(n)$ the scaling function of time associate to a map $T$.

In particular, on the different families of Definition 3.1, it is given by

$$
\Gamma_{T}(n)= \begin{cases}\log \left(\lambda^{n}\right) & \text { for the hyperbolic family of } T \\ \log n & \text { for the parabolic family of } T \\ 0 & \text { for the elliptic family of } T\end{cases}
$$

We shall concretely show that the difference (28) goes to zero with $N \rightarrow \infty$ in the strong topology over the Hilbert space $L_{\mu}^{2}\left(\mathbb{T}^{2}\right)$. More precisely, we have

\section{Theorem 1}

Let $\left(\mathcal{D}_{\mathcal{N}}, \tau_{\mathcal{N}}, \Theta_{\mathcal{N}}\right)$ be a sequence of discretized dynamical systems as defined 
in Section 3: for all $\gamma>1$,

$$
\forall f \in L_{\mu}^{\infty}\left(\mathrm{T}^{2}\right) \quad, \quad \operatorname{sim}_{\substack{j, N \rightarrow \infty \\ \Gamma_{T}(j)<\frac{\log N}{\gamma}}}\left(\Theta^{j}-\mathcal{J}_{\infty, \mathcal{N}} \circ \Theta_{\mathcal{N}}^{j} \circ \mathcal{J}_{\mathcal{N}, \infty}\right)(f)=0
$$

where the limit is in the strong topology over the Hilbert space $L_{\mu}^{2}\left(\mathrm{~T}^{2}\right)$.

The previous Theorem indicates that the time limit and the continuous limit do not commute in the parabolic and hyperbolic cases. In particular, the difference between the discretized dynamics and the continuous one can be made small by increasing $N$, while it becomes large beyond the time scale $\Gamma_{T}(j) \simeq \log N$. This phenomenon is the same as in quantum chaos and points to discretization of phase space (in the traditional semi-classical treatment of quantum systems), rather than to non-commutativity, as the source of the so-called logarithmic breaking time for hyperbolic systems. The constant $\gamma$ is a form factor, which reflects the fine structure of the dynamics: for instance, in the case of Quantum Cat Maps [14], $\gamma=2$.

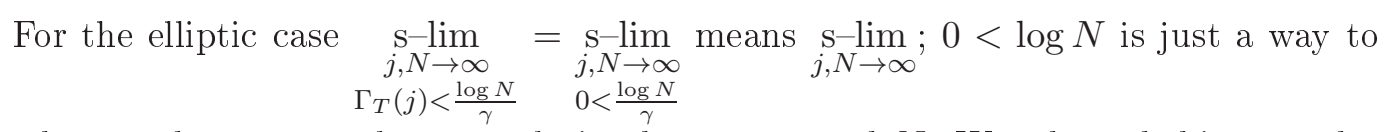
write that we do not consider any relation between $j$ and $N$. We adopted this, in order to have uniformity among the notations in the three different family of matrix action.

The constraint $j \leq C \log \mathcal{N}$ is typical of hyperbolic behaviour with Lyapunov exponent $\log \lambda$ and comes heuristically as follows: the expansion of an initial small distance $\delta$ can be exponential until the distance becomes the largest possible, namely $\delta \lambda^{T_{\mathrm{B}}} \simeq 1$ (on the torus). After discretization, the minimal distance gives $\delta=\frac{1}{N}$, therefore one estimates $T_{\mathrm{B}} \simeq \frac{\log N}{\log \lambda}$, which is called breaking time and sets the time-scale over which continuous and discretized dynamics mimic each other.

In quantum chaos, the semi-classical analysis leads to an estimate of $T_{\mathrm{B}}$ exactly as above; further, the logarithmic dependence on $\hbar$ of $T_{\mathrm{B}}$ is a signature of the hyperbolic character of the classical limit. Conversely, if the classical limit is regular (parabolic and elliptic case), then the time scale when quantum and classical behaviours are more or less indistinguishable goes in general as $\hbar^{-b}, b>0$.

The proof of Theorem 1 consists of several steps, among which the most important is a property, satisfied by our choice of Lattice States, which we shall call Dynamical Localization. We give a full proof that the Lattice States satisfies such property, since it represents a natural request that should be fulfilled by any consistent discretization/dediscretization (quantization/de-quantization) scheme; before giving the statement of the 
dynamical localization condition, let us introduce one more

\section{Definition 4.2}

We shall denote by $K_{N, n}(\boldsymbol{x}, \boldsymbol{y})$ the quantity

$$
K_{N, n}(\boldsymbol{x}, \boldsymbol{y}):=\left\langle C_{\mathcal{N}}(\boldsymbol{x}), W_{T, N}^{n} C_{\mathcal{N}}(\boldsymbol{y})\right\rangle=\left\langle U_{T}^{n}\left(\hat{\boldsymbol{x}}_{N}\right), \hat{\boldsymbol{y}}_{N}\right\rangle
$$

where $W_{T, N}^{j}$ is the unitary operator defined in $(27)$ and $\left\{\left|C_{\mathcal{N}}(\boldsymbol{x})\right\rangle\right\}$ is the set of LS of Definition 2.1 .

\section{Theorem 2 (Dynamical localization with $\left\{\left|C_{\mathcal{N}}(x)\right\rangle\right\}$ states)}

For every $\gamma>1$ and $d_{0}>0$, there exists $N_{0}=N_{0}\left(\gamma, d_{0}\right) \in \mathbb{N}^{+}$with the following property: if $N>N_{0}$ and $\Gamma_{T}(n)<\frac{\log N}{\gamma}$, then

$$
d_{\mathbb{T}^{2}}\left(T^{n}(\boldsymbol{x}), \boldsymbol{y}\right) \geqslant d_{0} \Longrightarrow K_{N, n}(\boldsymbol{x}, \boldsymbol{y})=0
$$

for all $\boldsymbol{x}, \boldsymbol{y} \in \mathbb{T}^{2}$, where $K_{N, n}(\boldsymbol{x}, \boldsymbol{y})$ are those of Definition 4.2 and the scaling function of time $\Gamma_{T}(n)$ has been introduced in Definition 4.1.

In analogy to the quantum case, dynamical localization is what one expects from a good choice of states suited the study of the continuous limit: in fact, it essentially amounts to asking that LS remain decently localized around the continuous trajectories while evolving with the corresponding discrete evolution. As we shall see this is the case only on time such that $\Gamma_{T}(n)<(\log N) / \gamma$. Informally, when $N \rightarrow \infty$, the quantities $K_{N, j}(\boldsymbol{x}, \boldsymbol{y})$ should behave as if $\mathcal{N}\left|K_{N, j}(\boldsymbol{x}, \boldsymbol{y})\right|^{2} \simeq \delta\left(T^{j}(\boldsymbol{x})-\boldsymbol{y}\right)$ and this is the content of next Proposition 4.1, that will be of use in Section 5.4.

This would make the discretization analogous to the notion of regular quantization described in Section V of [19]. Actually, with our choice of LS, the quantity $K_{N, j}(\boldsymbol{x}, \boldsymbol{y})$ is a Kronecker delta.

\section{Proposition 4.1}

Using the same notation of Theorem 2 we have that, for any given real number $\gamma>1$ and $f \in L_{\mu}^{\infty}\left(\mathrm{T}^{2}\right)$, it holds true:

$$
\lim _{\substack{n, N \rightarrow \infty \\ \Gamma_{T}(n)<\frac{\log N}{\gamma}}}\left\|\mathcal{N} \int_{\mathbb{T}^{2}} f(\boldsymbol{y})\left|K_{N, n}(\cdot, \boldsymbol{y})\right|^{2} \mu(\mathrm{d} \boldsymbol{y})-f\left(T^{n}(\cdot)\right)\right\|_{2}=0
$$

where $\|\cdot\|_{2}$ denotes the $L_{\mu}^{2}\left(\mathrm{~T}^{2}\right)$-norm. 


\section{Proof:}

The equation of the statement can be expressed in terms of the discretization-dediscretization operator $\mathcal{J}_{\mathcal{N}, \infty}$ and $\mathcal{J}_{\infty, \mathcal{N}}$ of Definition 2.2, the discrete evolution automorphism $\Theta_{\mathcal{N}}$ of Definition 3.3 and the continuous one $\Theta$ of Section 2, as follows:

$$
\lim _{\substack{n, N \rightarrow \infty \\ \Gamma_{T}(n)<\frac{\log N}{\gamma}}}\left\|\left(\Theta^{n}-\mathcal{J}_{\infty, \mathcal{N}} \circ \Theta_{\mathcal{N}}^{n} \circ \mathcal{J}_{\mathcal{N}, \infty}\right)(f)\right\|_{2}=0
$$

The last equation is proved in proof of Theorem 1 (see (44)).

In order to prove Theorem 2, we need the following auxiliary result.

\section{Proposition 4.2}

Resorting to the distance (5), $\hat{\boldsymbol{x}}_{N}$ of Definition 2.1, $U_{T}$ of $(26)$ and $(\lambda, \beta, J, \eta)$ used in Propositions 3.1-3.3, the following three statements hold:

For $\boldsymbol{x} \in \mathbb{T}^{2}$ and $n \in \mathbb{N}^{+}$

1) if $T$ is hyperbolic and $N>\widetilde{N}_{\text {hyp }}(n):=\sqrt{2} \frac{\lambda^{n}}{\sin \beta}$

$$
\text { then } d_{\mathbb{T}^{2}}\left(T^{p}(\boldsymbol{x}), \frac{U_{T}^{p}\left(\hat{\boldsymbol{x}}_{N}\right)}{N}\right) \leqslant \frac{\widetilde{N}_{\mathrm{hyp}}(n)}{2 N} \quad, \quad \forall p \leqslant n ;
$$

2) if $T$ is parabolic and $N>\widetilde{N}_{\text {par }}(n):=\sqrt{2}(2 n J+1)$

$$
\text { then } d_{\mathbb{T}^{2}}\left(T^{p}(\boldsymbol{x}), \frac{U_{T}^{p}\left(\hat{\boldsymbol{x}}_{N}\right)}{N}\right) \leqslant \frac{\widetilde{N}_{\mathrm{par}}(n)}{2 N} \quad, \quad \forall p \leqslant n ;
$$

3) if $T$ is elliptic and $N>\widetilde{N}_{\text {ell }}:=\sqrt{2} \eta$

$$
\text { then } d_{\mathbb{T}^{2}}\left(T^{p}(\boldsymbol{x}), \frac{U_{T}^{p}\left(\hat{\boldsymbol{x}}_{N}\right)}{N}\right) \leqslant \frac{\widetilde{N}_{\mathrm{ell}}}{2 N} \quad, \quad \forall p \leqslant n .
$$

\section{Proof:}

For every real number $t$, we have $0 \leqslant\langle N t+1 / 2\rangle=N t+1 / 2-\lfloor N t+1 / 2\rfloor<1$, so that $\left|t-\frac{\lfloor N t+1 / 2\rfloor}{N}\right| \leqslant \frac{1}{2 N}, \forall t \in \mathbb{R}$. From (6) in Definition 2.1, we derive

$$
d_{\mathbb{T}^{2}}\left(\boldsymbol{x}, \frac{\hat{\boldsymbol{x}}_{N}}{N}\right) \leqslant \frac{1}{\sqrt{2} N} \quad, \quad \forall \boldsymbol{x} \in \mathbb{T}^{2}
$$

Let us start by proving the first statement, being the other very similar to it. Using the definition of $U_{T}$ given in (26), we write

$$
\left\|T^{p}(\boldsymbol{x})-\frac{U_{T}^{p}\left(\hat{\boldsymbol{x}}_{N}\right)}{N}\right\|_{\mathbb{R}^{2}}=\left\|T^{p}(\boldsymbol{x})-T^{p}\left(\frac{\hat{\boldsymbol{x}}_{N}}{N}\right)\right\|_{\mathbb{R}^{2}}=\left\|T^{p}\left(\boldsymbol{x}-\frac{\hat{\boldsymbol{x}}_{N}}{N}\right)\right\|_{\mathbb{R}^{2}},
$$


where in the latter equality we applied the linearity of $T(\cdot)$. As (16) was the maximum allowed spreading for the unit ball $B_{T}(0)$ under the action of $n$ power of the matrix $T$, now we have

$$
\left\|T^{p}\left(\boldsymbol{x}-\frac{\hat{\boldsymbol{x}}_{N}}{N}\right)\right\|_{\mathbb{R}^{2}} \leqslant \frac{\lambda^{p}}{\sin \beta}\left\|\boldsymbol{x}-\frac{\hat{\boldsymbol{x}}_{N}}{N}\right\|_{\mathbb{R}^{2}} \leqslant \frac{1}{\sqrt{2} N} \frac{\lambda^{n}}{\sin \beta},
$$

indeed $p \leqslant n$ and we applied (33) together with Remark 2.2.i. In order to replace the first norm in (34) with the toral distance, we apply once more the same Remark 2.2.i, providing that $\frac{1}{\sqrt{2} N} \frac{\lambda^{n}}{\sin \beta} \leqslant \frac{1}{2}$, that is $N \geqslant N_{\text {hyp }}(n)$.

The other statement (31-32) are proved in the same way, substituting in (35) the right expression for the diameters, given for parabolic and elliptic case from (22), respectively (24).

\section{Proof of Theorem 2 :}

Using the definition of $\left\{\left|C_{\mathcal{N}}(\boldsymbol{x})\right\rangle\right\}$ in (7), we easily compute

$$
\left\langle C_{\mathcal{N}}(\boldsymbol{x}) \mid W_{T, N}^{n} C_{\mathcal{N}}(\boldsymbol{y})\right\rangle=\left\langle\hat{\boldsymbol{x}}_{N} \mid U_{T}^{-n}\left(\hat{\boldsymbol{y}}_{N}\right)\right\rangle=\delta_{U_{T}^{n}\left(\hat{\boldsymbol{x}}_{N}\right), \hat{\boldsymbol{y}}_{N}}^{(N)} .
$$

Using the triangular inequality, we get

$$
\begin{aligned}
d_{\mathbb{T}^{2}}\left(\frac{U_{T}^{n}\left(\hat{\boldsymbol{x}}_{N}\right)}{N}, \frac{\hat{\boldsymbol{y}}_{N}}{N}\right) \geqslant d_{\mathbb{T}^{2}}\left(T^{n}(\boldsymbol{x}), \boldsymbol{y}\right)- & \\
& \quad-d_{\mathbb{T}^{2}}\left(T^{n}(\boldsymbol{x}), \frac{U_{T}^{n}\left(\hat{\boldsymbol{x}}_{N}\right)}{N}\right)-d_{\mathbb{T}^{2}}\left(\frac{\hat{\boldsymbol{y}}_{N}}{N}, \boldsymbol{y}\right) .
\end{aligned}
$$

Now we split the proof and we begin by focusing on the

\section{Hyperbolic case:}

Since $d_{\mathbb{T}^{2}}\left(T^{n}(\boldsymbol{x}), \boldsymbol{y}\right) \geqslant d_{0}$ by hypothesis, using (33) of proof of Proposition 4.2 and (30), that is

$$
N>\tilde{N}_{\text {hyp }}(n) \Longrightarrow d_{\mathbb{T}^{2}}\left(T^{n}(\boldsymbol{x}), \frac{U_{T}^{n}\left(\hat{\boldsymbol{x}}_{N}\right)}{N}\right) \leqslant \frac{1}{\sqrt{2} N} \frac{\lambda^{n}}{\sin \beta},
$$

we can derive from (37) that $d_{\mathbb{T}^{2}}\left(\frac{U_{T}^{n}\left(\hat{\boldsymbol{x}}_{N}\right)}{N}, \frac{\hat{\boldsymbol{y}}_{N}}{N}\right) \geqslant d_{0}-\frac{1}{\sqrt{2} N} \frac{\lambda^{n}}{\sin \beta}-\frac{1}{\sqrt{2} N}$.

The r.h.s. of the previous inequality can always be made strictly larger than zero,

$$
d_{\mathbb{T}^{2}}\left(\frac{U_{T}^{n}\left(\hat{\boldsymbol{x}}_{N}\right)}{N}, \frac{\hat{\boldsymbol{y}}_{N}}{N}\right)>0
$$


by choosing an $N$ larger than

$$
N_{\mathrm{M}}(n)=\max \left\{\frac{1}{d_{0} \sqrt{2}}\left(1+\frac{\lambda^{n}}{\sin \beta}\right), \widetilde{N}_{\text {hyp }}(n)=\sqrt{2} \frac{\lambda^{n}}{\sin \beta}\right\},
$$

so that the condition on the 1.h.s. of (38) is also satisfied. From (36) and (39), we have

$$
N>N_{\mathrm{M}}(n) \quad \Longrightarrow \quad\left\langle C_{\mathcal{N}}(\boldsymbol{x}) \mid W_{T, N}^{n} C_{\mathcal{N}}(\boldsymbol{y})\right\rangle=0
$$

Indeed, if the toral distance between two grid points $\left(\hat{\boldsymbol{z}}_{N}, \hat{\boldsymbol{w}}_{N}\right)$ is different from zero, they can not by equal $(\bmod N)$ and so the periodic Kronecker delta in (36) vanishes.

Since the (non-decreasing) function $N_{\mathrm{M}}(n)$ in (40) is eventually bounded by $\lambda^{\gamma n}(\gamma$ being strictly greater than one), we define $\bar{n}$ as the time when $N_{\mathrm{M}}(\bar{n})=\lambda^{\gamma \bar{n}}=: N_{0}$, and choose $N>N_{0}$. Thus, if $0<n<\bar{n}$, then $N>N_{0}=N_{\mathrm{M}}(\bar{n})>N_{\mathrm{M}}(n)$, whereas if $\bar{n} \leqslant n<\frac{1}{\gamma} \frac{\log N}{\log \lambda}$, then $N>\lambda^{\gamma n}>N_{\mathrm{M}}(n)$ and (41) holds for all $0<n<\frac{1}{\gamma} \frac{\log N}{\log \lambda}$, that is $\Gamma_{T}(n)<\frac{\log N}{\gamma}$ as in the statement.

\section{Parabolic case:}

Using now (31), that is

$$
N>\widetilde{N}_{\mathrm{par}}(n) \Longrightarrow d_{\mathbb{T}^{2}}\left(T^{n}(\boldsymbol{x}), \frac{U_{T}^{n}\left(\hat{\boldsymbol{x}}_{N}\right)}{N}\right) \leqslant \frac{1}{\sqrt{2} N}(2 n J+1),
$$

we earn from $(37)$ that $d_{\mathbb{T}^{2}}\left(\frac{U_{T}^{n}\left(\hat{\boldsymbol{x}}_{N}\right)}{N}, \frac{\hat{\boldsymbol{y}}_{N}}{N}\right) \geqslant d_{0}-\frac{1}{\sqrt{2} N}(2 n J+1)-\frac{1}{\sqrt{2} N} \cdot$

The r.h.s. of the previous inequality can be made strictly larger than zero, by choosing an $N$ larger than

$$
N_{\mathrm{M}}(n)=\max \left\{\frac{\sqrt{2}}{d_{0}}(n J+1), \tilde{N}_{\mathrm{par}}(n)=\sqrt{2}(2 n J+1)\right\},
$$

so that the condition on the 1.h.s. of (42) is also satisfied. Reasoning as for the hyperbolic case, we conclude that (41) still hold true in this case and we choose $n^{\gamma}$ as bounding function of the (non-decreasing) $N_{\mathrm{M}}(n)$ of (43).

Finally, as for the hyperbolic case, we define $\bar{n}$ as the time when $N_{\mathrm{M}}(\bar{n})=\bar{n}^{\gamma} \Rightarrow N_{0}$, and choose $N>N_{0}$. Thus, if $0<n<\bar{n}$, then $N>N_{0}=N_{\mathrm{M}}(\bar{n})>N_{\mathrm{M}}(n)$, whereas if $\bar{n} \leqslant n<N^{\frac{1}{\gamma}}$, then $N>n^{\gamma}>N_{\mathrm{M}}(n)$ and (41) holds for all $0<n<N^{\frac{1}{\gamma}}$, that is $\Gamma_{T}(n)<\frac{\log N}{\gamma}$ as in the statement. 


\section{Elliptic case:}

The same strategy adopted in the previous two cases, lead now us to define a new $N_{\mathrm{M}}$, independent of $n$, given by $N_{\mathrm{M}}=\max \left\{\frac{1}{d_{0} \sqrt{2}}(\eta+1), \widetilde{N}_{\text {ell }}(n)=\eta \sqrt{2}\right\}$; thus, for $N>N_{\mathrm{M}}$, the periodic Kronecker delta in (36) vanishes.

The absence of relation between $N$ and $n$, for $N>N_{\mathrm{M}}$, is expressed in the relation $\Gamma_{T}=0<\frac{\log N}{\gamma}$, always true for all $N$.

We are finally in position to conclude with

\section{Proof of Theorem 1:}

We will concentrate on the case of continuous $f$, that is $f \in \mathcal{C}^{0}\left(\mathrm{~T}^{2}\right)\left(\subset L_{\mu}^{2}\left(\mathrm{~T}^{2}\right)\right)$; the extension to essentially bounded $f$ is straightforward and can be realized by applying Lusin's Theorem [23,24,26], as the reader can check in [21].

Let $f \in \mathcal{C}^{0}\left(\mathrm{~T}^{2}\right)$ and $\mathrm{Op}_{j, N}(f):=\left(\Theta^{j}-\mathcal{J}_{\infty, \mathcal{N}} \circ \Theta_{\mathcal{N}}^{j} \circ \mathcal{J}_{\mathcal{N}, \infty}\right)(f)$ : notice that $\mathrm{Op}_{j, N}(f)$ is a multiplication operator on $L_{\mu}^{2}\left(\mathbb{T}^{2}\right)$, but also an $L_{\mu}^{\infty}\left(\mathbb{T}^{2}\right)\left(\right.$ and thus also an $\left.L_{\mu}^{2}\left(\mathbb{T}^{2}\right)\right)$ function. According to (29), we must show that

$$
\forall g \in L_{\mu}^{2}\left(\mathrm{~T}^{2}\right) \quad, \quad \lim _{\substack{j, N \rightarrow \infty \\ \Gamma_{T}(j)<\frac{\log N}{\gamma}}}\left\|\mathrm{Op}_{j, N}(f) g\right\|_{2}=0
$$

Using Schwartz's inequality first with $g$ in the class of simple functions and then using their density in $L_{\mu}^{2}\left(\mathbb{T}^{2}\right)$, we have just to show that

$$
\lim _{\substack{j, N \rightarrow \infty \\ \Gamma_{T}(j)<\frac{\log N}{\gamma}}}\left\|\mathrm{Op}_{j, N}(f)\right\|_{2}=0
$$

In [21] it is shown that

$$
\left\|\mathrm{Op}_{j, N}(f)\right\|_{2}^{2}=\omega_{\mu}\left(|f|^{2}\right)+\tau_{\mathcal{N}}\left[\mathcal{J}_{\mathcal{N}, \infty}(f)^{*} \mathcal{J}_{\mathcal{N}, \infty}(f)\right]-2 \operatorname{Re}\left(I_{j, N}(f)\right)
$$

with

$$
\begin{aligned}
I_{j, N}(f) & :=\tau_{\mathcal{N}}\left[\left(\mathcal{J}_{\mathcal{N}, \infty} \circ \Theta^{j}\right)(f)^{*}\left(\Theta_{\mathcal{N}}^{j} \circ \mathcal{J}_{\mathcal{N}, \infty}\right)(f)\right] \\
& =\mathcal{N} \int_{\mathbb{T}^{2}} \mu(\mathrm{d} \boldsymbol{x}) \int_{\mathbb{T}^{2}} \mu(\mathrm{d} \boldsymbol{y}) \overline{f(\boldsymbol{y})} f\left(T^{j}(\boldsymbol{x})\right)\left|\left\langle C_{\mathcal{N}}(\boldsymbol{x}), W_{T, N}^{j} C_{\mathcal{N}}(\boldsymbol{y})\right\rangle\right|^{2}
\end{aligned}
$$

and that $\tau_{\mathcal{N}}\left[\mathcal{J}_{\mathcal{N}, \infty}(f)^{*} \mathcal{J}_{\mathcal{N}, \infty}(f)\right] \longrightarrow \omega_{\mu}\left(|f|^{2}\right)$ for large $N$; so now the strategy is to prove that also $I_{j, N}(f)$ goes to $\omega_{\mu}\left(|f|^{2}\right)=\int_{\mathbb{T}^{2}} \mu(\mathrm{d} \boldsymbol{x})|f(\boldsymbol{x})|^{2}$ when $j, N \rightarrow \infty$ with 
$\Gamma_{T}(j)<\frac{\log N}{\gamma}$. We want to prove that the difference

$$
\begin{aligned}
& \left.\left|I_{j, N}(f)-\int_{\mathbb{T}^{2}} \mu(\mathrm{d} \boldsymbol{y})\right| f(\boldsymbol{y})\right|^{2} \mid \\
& =\left.\left|\int_{\mathbb{T}^{2}} \mu(\mathrm{d} \boldsymbol{x}) \int_{\mathbb{T}^{2}} \mu(\mathrm{d} \boldsymbol{y}) \overline{f(\boldsymbol{y})}\left(f\left(T^{j}(\boldsymbol{x})\right)-f(\boldsymbol{y})\right) \mathcal{N}\right|\left\langle C_{\mathcal{N}}(\boldsymbol{x}), W_{T, N}^{j} C_{\mathcal{N}}(\boldsymbol{y})\right\rangle\right|^{2} \mid
\end{aligned}
$$

is negligible for large $N$ : selecting a ball $B\left(T^{j}(\boldsymbol{x}), d_{0}\right)$, one derives

$$
\begin{aligned}
& \leq\left.\left|\int_{\mathbb{T}^{2}} \mu(\mathrm{d} \boldsymbol{x}) \int_{B\left(T^{j}(\boldsymbol{x}), d_{0}\right)} \mu(\mathrm{d} \boldsymbol{y}) \overline{f(\boldsymbol{y})}\left(f\left(T^{j}(\boldsymbol{x})\right)-f(\boldsymbol{y})\right) \mathcal{N}\right|\left\langle C_{\mathcal{N}}(\boldsymbol{x}), W_{T, N}^{j} C_{\mathcal{N}}(\boldsymbol{y})\right\rangle\right|^{2} \mid \\
& +\left.\left|\int_{\mathbb{T}^{2}} \mu(\mathrm{d} \boldsymbol{x}) \int_{\mathbb{T}^{2} \backslash B\left(T^{j}(\boldsymbol{x}), d_{0}\right)} \mu(\mathrm{d} \boldsymbol{y}) \overline{f(\boldsymbol{y})}\left(f\left(T^{j}(\boldsymbol{x})\right)-f(\boldsymbol{y})\right) \mathcal{N}\right|\left\langle C_{\mathcal{N}}(\boldsymbol{x}), W_{T, N}^{j} C_{\mathcal{N}}(\boldsymbol{y})\right\rangle\right|^{2} \mid .
\end{aligned}
$$

Applying the mean value theorem in the first double integral, we get that $\exists \boldsymbol{c} \in B\left(T^{j}(\boldsymbol{x}), d_{0}\right)$ such that

$$
\begin{aligned}
& \left.\left|I_{j, N}(f)-\int_{\mathbb{T}^{2}} \mu(\mathrm{d} \boldsymbol{y})\right| f(\boldsymbol{y})\right|^{2} \mid \\
& \leq \int_{\mathbb{T}^{2}} \mu(\mathrm{d} \boldsymbol{x})\left|\overline{f(\boldsymbol{c})}\left(f\left(T^{j}(\boldsymbol{x})\right)-f(\boldsymbol{c})\right)\right| \int_{B\left(T^{j}(\boldsymbol{x}), d_{0}\right)} \mu(\mathrm{d} \boldsymbol{y}) \mathcal{N}\left|\left\langle\left(W_{T, N}^{*}\right)^{j} C_{\mathcal{N}}(\boldsymbol{x}), C_{\mathcal{N}}(\boldsymbol{y})\right\rangle\right|^{2} \\
& \quad+2\|f\|_{0}^{2} \int_{\mathbb{T}^{2}} \mu(\mathrm{d} \boldsymbol{x}) \int_{\mathbb{T}^{2} \backslash B\left(T^{j}(\boldsymbol{x}), d_{0}\right)} \mu(\mathrm{d} \boldsymbol{y}) \mathcal{N}\left|\left\langle C_{\mathcal{N}}(\boldsymbol{x}), W_{T, N}^{j} C_{\mathcal{N}}(\boldsymbol{y})\right\rangle\right|^{2},
\end{aligned}
$$

where we used the uniform norm $\|\cdot\|_{0}$, indeed $f \in \mathcal{C}^{0}\left(\mathrm{~T}^{2}\right)$. Finally, using completeness and normalization (Properties 2.1), we arrive at the upper bound

$$
\leq\|f\|_{0} \sup _{\substack{\boldsymbol{z} \in \mathbb{T}^{2} \\ \boldsymbol{c} \in B\left(\boldsymbol{z}, d_{0}\right)}}|(f(\boldsymbol{z})-f(\boldsymbol{c}))|+2\|f\|_{0}^{2} \quad \mathcal{N} \sup _{\substack{\boldsymbol{x} \in \mathbb{T}^{2} \\ \boldsymbol{y} \notin B\left(T^{j}(\boldsymbol{x}), d_{0}\right)}}\left|\left\langle C_{\mathcal{N}}(\boldsymbol{x}), W_{T, N}^{j} C_{\mathcal{N}}(\boldsymbol{y})\right\rangle\right|^{2} .
$$

By uniform continuity, the first term can be made arbitrarily small, provided we choose $d_{0}$ small enough. For the second integral, we use Theorem 2, which provides us with $N_{0}=N_{0}\left(\gamma, d_{0}\right)$ depending on the same $d_{0}$, such that the second term vanishes for all $N>N_{0}$ and for all $j$ such that $\Gamma_{T}(j)<\frac{\log N}{\gamma}$.

\section{Dynamical Entropy on Discrete Systems}

Dealing with hyperbolic systems, one expects the instability proper to the presence of a positive Lyapunov exponent to correspond to some degree of unpredictability of the 
dynamics: classically, the metric entropy of Kolmogorov-Sinai provides the link [27].

\subsection{A classical one: Kolmogorov-Sinai metric entropy}

For continuous classical systems $(\mathcal{X}, \mu, T)$ such as those introduced in Section 2, the construction of the dynamical entropy of Kolmogorov-Sinai is based on subdividing $\mathcal{X}$ into measurable disjoint subsets $\left\{E_{\ell}\right\}_{\ell=1,2, \cdots, D}$ such that $\bigcup_{\ell} E_{\ell}=\mathcal{X}$ which form finite partitions (coarse graining $\mathrm{s}) \mathcal{E}$.

Under the action of dynamical maps $T$ in (1), any given partition $\mathcal{E}$ evolves into $T^{-j}(\mathcal{E})$ with atoms $T^{-j}\left(E_{\ell}\right)=\left\{\boldsymbol{x} \in \mathcal{X}: T^{j}(\boldsymbol{x}) \in E_{\ell}\right\}$; one can then form finer partitions $\mathcal{E}_{[0, n-1]}:=\bigvee_{j=0}^{n-1} T^{j}(\mathcal{E})$ whose atoms $E_{i_{0} i_{1} \cdots i_{n-1}}:=\bigcap_{j=0}^{n-1} T^{-j} E_{i_{j}}$ have volumes $\mu_{i_{0} i_{1} \cdots i_{n-1}}:=\mu\left(E_{i_{0} i_{1} \cdots i_{n-1}}\right)$.

\section{Definitions 5.1}

1) We shall set $\boldsymbol{i}=\left\{i_{0} i_{1} \cdots i_{n-1}\right\}$ and denote by $\Omega_{D}^{n}$ the set of $D^{n}$ n_tuples with $i_{j}$ taking values in $\{1,2, \cdots, D\}$.

2) The symbol $\hat{\boldsymbol{\imath}}$ will indicate the string $\hat{\boldsymbol{\imath}}:=\left\{i_{n-1} i_{n-2} \cdots i_{1} i_{0}\right\} \in \Omega_{D}^{n}$; the two string $\boldsymbol{i}$ and $\hat{\boldsymbol{\imath}}$ are related by $i_{j}=\hat{\imath}_{n-1-j}, \forall j \in\{0, \ldots, n-1\}$.

The atoms of the partitions $\mathcal{E}_{[0, n-1]}$ describe segments of trajectories up to time $n$ encoded by the atoms of $\mathcal{E}$ that are traversed at successive times; the volumes $\mu_{\boldsymbol{i}}=\mu\left(E_{\boldsymbol{i}}\right)$ corresponds to probabilities for the system to belong to the atoms $E_{i_{0}}, E_{i_{1}}, \cdots, E_{i_{n-1}}$ at successive times $0 \leqslant j \leqslant n-1$. The richness in diverse trajectories, that is the degree of irregularity of the motion (as seen with the accuracy of the given coarse-graining) correspond intuitively to our idea of "complexity" and can be measured by the Shannon entropy [16] $S_{\mu}\left(\mathcal{E}_{[0, n-1]}\right):=-\sum_{\boldsymbol{i} \in \Omega_{D}^{n}} \mu_{\boldsymbol{i}} \log \mu_{\boldsymbol{i}}$.

On the long run, the partition $\mathcal{E}$ attributes to the dynamics an entropy per unit time-step $h_{\mu}(T, \mathcal{E}):=\lim _{n \rightarrow \infty} \frac{1}{n} S_{\mu}\left(\mathcal{E}_{[0, n-1]}\right)$.

This limit is well defined [7] and the "average entropy production" $h_{\mu}(T, \mathcal{E})$ measure how predictable the dynamics is on the coarse grained scale provided by the finite partition $\mathcal{E}$. To remove the dependence on $\mathcal{E}$, the $\mathrm{KS}$ entropy $h_{\mu}(T)$ of $(\mathcal{X}, \mu, T)$ is defined as the supremum over all finite measurable partitions $[7,16] h_{\mu}(T):=\sup _{\mathcal{E}} h_{\mu}(T, \mathcal{E})$.

\subsection{Dynamics and Information in the Quantum Setting}

From an algebraic point of view, the difference between a "quantum" triplet $(\mathcal{M}, \omega, \Theta)$ describing a quantum dynamical system and classical triplets like $\left(L_{\mu}^{\infty}\left(\mathrm{T}^{2}\right), \omega_{\mu}, \Theta\right)$ of 
Section 2 or $\left(\mathcal{D}_{\mathcal{N}}, \tau_{\mathcal{N}}, \Theta_{\mathcal{N}}\right)$ of Section 2.1 is that $\omega$ and $\Theta$ are now a $\Theta$-invariant state, respectively an automorphism over a non-commutative $\left(\mathrm{C}^{*}\right.$ or Von Neumann) algebra of operators $\mathcal{M}[11]$.

- In standard quantum mechanics the algebra $\mathcal{M}$ is the von Neumann algebra $B(\mathcal{H})$ of all bounded linear operators on a suitable Hilbert space $\mathcal{H}$. If $\mathcal{H}$ has finite dimension $D, \mathcal{M}$ is the algebra of $D \times D$ matrices.

- The typical states $\omega$ are density matrices $\rho$, namely operators with positive eigenvalues $\rho_{\ell}$ such that $\operatorname{Tr}(\rho)=\sum_{\ell} \rho_{\ell}=1$. Given the state $\rho$, the mean value of any observable $X \in B(\mathcal{H})$ is given by $\rho(X):=\operatorname{Tr}(\rho X)$.

- The $\rho_{\ell}$ of previous point are interpreted as probabilities of finding the system in the corresponding eigenstates. The uncertainty prior to the measurement is measured by the Von Neumann entropy of $\rho$ given by $H(\rho):=-\operatorname{Tr}(\rho \log \rho)=-\sum_{\ell} \rho_{\ell} \log \rho_{\ell}$.

- The usual dynamics on $\mathcal{M}$ is of the form $\Theta(X)=U X U^{*}$, where $U$ is a unitary operator. If one has a Hamiltonian operator that generates the continuous group $U_{t}=\exp i t H / \hbar$ then $U:=U_{t=1}$ and the time-evolution is discretized by considering powers $U^{j}$.

The idea behind the notion of dynamical entropy is that information can be obtained by repeatedly observing a system in the course of its time evolution. Due to the uncertainty principle, or, in other words, to non-commutativity, if observations are intended to gather information about the intrinsic dynamical properties of quantum systems, then non-commutative extensions of the KS-entropy ought first to decide whether quantum disturbances produced by observations have to be taken into account or not.

Concretely, let us consider a quantum system described by a density matrix $\rho$ acting on a Hilbert space $\mathcal{H}$. Via the wave packet reduction postulate, generic measurement processes may reasonably well be described by finite sets $\mathcal{Y}=\left\{y_{0}, y_{1}, \ldots, y_{D-1}\right\}$ of bounded operators $y_{j} \in \mathcal{B}(\mathcal{H})$ such that $\sum_{j} y_{j}^{*} y_{j}=\mathbb{1}$. These sets are called partitions of unity (p.u., for sake of shortness) and describe the change in the state of the system caused by the corresponding measurement process:

$$
\rho \longmapsto \Gamma_{\mathcal{Y}}^{*}(\rho):=\sum_{j} y_{j} \rho y_{j}^{*}
$$

It looks rather natural to rely on partitions of unity to describe the process of collecting information through repeated observations of an evolving quantum system [18]. 
Our intention is now to introduce a quantum dynamical entropy [19], based and constructed by means of CS, and apply it to our families of discretized toral automorphisms. We will show that this quantity does reduce to the Kolmogorov-Sinai invariant, but only for time scales bounded by the logarithm of the discretization parameter $N$.

It is worth mention that the same result has been proved in [14] for two differents quantum dynamical entropies (called ALF- and CNT-entropy) applied to finite dimensional quantum counterparts of the hyperbolic family of UMG that we have considered within this paper. The only hypothesis used in [14] to get the above mentioned result, consisted of a dynamical localization property analogous to the one we proved in Theorem 2.

As a consequence, the same results of [14], that is the convergence of ALF- and CNT-entropy to the KS one, can be obtained also in the present framework.

\subsection{CS Quantum Entropies}

In order to make the description of a quantum system closer to that of a classical one, the most useful tool consists in using CS. The quantum measurement process itself can be depicted in terms of CS in such a way that classical property can be recovered in the semi-classical limit.

Let $(\mathcal{M}, \omega, \Theta)$ be a (finite dimensional) quantum dynamical system as the ones introduced in Section 5.2, with $\mathcal{N}$ denoting the dimension of its Hilbert space $\mathcal{H}$, and $(\mathcal{X}, \mu, T)$ be its classical counterpart, the latter endowed with a classical partition $\mathcal{E}=$ $\left\{E_{\ell}\right\}_{\ell=1,2, \cdots, D}$ on it (see Section 5.1). Introduce on such a system a family of Coherent States endowed with properties 2.1.

The map

$$
\mathcal{I}(C)(\rho):=\mathcal{N} \int_{C}\left|C_{\mathcal{N}}(\boldsymbol{x})\right\rangle\left\langle C_{\mathcal{N}}(\boldsymbol{x})|\rho| C_{\mathcal{N}}(\boldsymbol{x})\right\rangle\left\langle C_{\mathcal{N}}(\boldsymbol{x})\right| \mu(\mathrm{d} \boldsymbol{x})
$$

for a measurable subset $C \subset \mathcal{X}$ and an operator $\rho$, is called an instrument [19]. The map $\rho \longmapsto \mathcal{I}(C)(\rho)$ describe the change in the state $\rho$ of the system caused by a $C$-dependent measurement process (compare with (45)).

If we take the expectation of $\mathcal{I}(C)(\rho)$, that is $\mu^{(\rho)}(C):=\omega[\mathcal{I}(C)(\rho)]$, we get the probability that a measurement on the system by the instrument (46) give values in $C$, when the pre-measurement state is $\rho$. If we wonder what is the probability that several measure, taken stroboscopically at times $t_{0}=0, t_{1}=1, \ldots, t_{n-1}=n-1$, give values 
in $E_{i_{0}}, E_{i_{1}}, \ldots, E_{i_{n-1}}$, we have to compose the instrument action (46) with the temporal evolution depicted in Section 5.2, obtaining

$$
\begin{aligned}
\mathcal{P}_{i_{0}, i_{1}, \ldots, i_{n-1}}^{\mathrm{CS}} & :=\mu_{t_{0}, t_{1}, \ldots, t_{n-1}}^{(\rho)}\left(E_{i_{0}} \times E_{i_{1}} \times \cdots \times E_{i_{n-1}}\right)= \\
& =\omega\left[\mathcal{I}\left(E_{i_{n-1}}\right) \circ \Theta \circ \mathcal{I}\left(E_{i_{n-2}}\right) \circ \Theta \circ \cdots \circ \mathcal{I}\left(E_{i_{1}}\right) \circ \Theta \circ \mathcal{I}\left(E_{i_{0}}\right)(\rho)\right]
\end{aligned}
$$

Using in (47) the expression for the dynamical evolution $\Theta(X)=U X U^{*}$ together with (46), and replacing the expectation $\omega$ with the trace, (see Section 5.2), we obtain

$$
\begin{aligned}
\mathcal{P}_{\boldsymbol{i}}^{\mathrm{CS}}= & \mathcal{P}_{i_{0}, i_{1}, \ldots, i_{n-1}}^{\mathrm{CS}}=\mathcal{N}^{n} \int_{E_{i_{0}}} \int_{E_{i_{1}}} \cdots \int_{E_{i_{n-1}}}\left\langle C_{\mathcal{N}}\left(\boldsymbol{x}_{0}\right)|\rho| C_{\mathcal{N}}\left(\boldsymbol{x}_{0}\right)\right\rangle \times \\
& \times \prod_{j=1}^{n-1}\left[\left|\left\langle C_{\mathcal{N}}\left(\boldsymbol{x}_{j}\right)|U| C_{\mathcal{N}}\left(\boldsymbol{x}_{j-1}\right)\right\rangle\right|^{2}\right] \mu\left(\mathrm{d} \boldsymbol{x}_{0}\right) \mu\left(\mathrm{d} \boldsymbol{x}_{1}\right) \cdots \mu\left(\mathrm{d} \boldsymbol{x}_{n-1}\right)
\end{aligned}
$$

where we have used the normalization property for the state $\left|C_{\mathcal{N}}\left(\boldsymbol{x}_{n-1}\right)\right\rangle$ and the notation given in Definition 5.1 for the strings $\boldsymbol{i}$.

This quantities can be seen as quantum analogue to the classical probability $\mu_{i}$ of Section 5.1 (in particular they sum up to one) and thus can be used in computing a Shannon entropy, depending on the given dynamics $U$, the instrument (46), the classical partition $\mathcal{E}$, the initial state $\rho$ and the considered time of measuring $n$, whose expression is

$$
S(U, \mathcal{I}, \mathcal{E}, \rho, n):=-\sum_{i \in \Omega_{D}^{n}} \mathcal{P}_{i}^{C S} \log \mathcal{P}_{i}^{C S}
$$

The CS quantum entropy [19] is defined as the "average production" on the long run of last quantity

$$
H(U, \mathcal{I}, \mathcal{E}, \rho):=\lim _{n \rightarrow \infty} \frac{1}{n} S(U, \mathcal{I}, \mathcal{E}, \rho, n)
$$

and it is decomposable in two component. The first, called measurement CS quantum entropy, is independent on the dynamics, originated by the pure measurement process, and obtained by replacing the unitary operator $U$ in $(50)$ with the identity on $\mathcal{H}$; its expression is

$$
H_{\text {meas }}(\mathcal{I}, \mathcal{E}, \rho):=H\left(\mathbb{1}_{\mathcal{N}}, \mathcal{I}, \mathcal{E}, \rho\right)
$$

The second amount to the remaining part

$$
H_{\text {dyn }}(U, \mathcal{I}, \mathcal{E}, \rho)=H(U, \mathcal{I}, \mathcal{E}, \rho)-H_{\text {meas }}(\mathcal{I}, \mathcal{E}, \rho)
$$


and is supposed to incorporate the dynamic dependence.

\subsection{CS Entropies for discrete classical systems}

The quantum entropy of last section can be seen as an algebraic quantity, and does need nothing more that the algebraic framework already developed in Sections 2-4, in order to be defined. In particular, we are going to estimate the CS entropy of discrete classical systems $\left(\mathcal{D}_{\mathcal{N}}, \tau_{\mathcal{N}}, \Theta_{\mathcal{N}}\right)$, using the Lattice States of Definition 2.1

Theorem 3 : Let $\left(\mathrm{T}^{2}, \mu, T\right)$ be the classical dynamical system of Section 2, which is the continuous limit of a sequence of finite dimensional discrete $d y$ namical systems $\left(\mathcal{D}_{\mathcal{N}}, \tau_{\mathcal{N}}, \Theta_{\mathcal{N}}\right)$. If

1. $W_{T, N}$ is the unitary evolution operator of $(27)$;

2. I in the instrument (46) constructed with the LS of Definition 2.1;

3. $\mathcal{E}=\left\{E_{0}, E_{1}, \ldots, E_{D-1}\right\}$ is a finite measurable partition of $\mathbb{T}^{2}$;

4. $\rho$ is the tracial state $\frac{1}{\mathcal{N}} \mathbb{1}_{\mathcal{N}}$;

then there exists an $\alpha$ such that

$$
\lim _{\substack{n, N \rightarrow \infty \\ n<\alpha \log N}} \frac{1}{n}\left|S\left(W_{T, N}, \mathcal{I}, \mathcal{E}, \rho, n\right)-S_{\mu}\left(\mathcal{E}_{[0, n-1]}\right)\right|=0
$$

In order to prove Theorem 3, we need the following auxiliary result.

\section{Lemma 5.1}

Suppose to have a sequence $\left\{g_{N}\right\}$ of $L_{\mu}^{2}\left(\mathbb{T}^{2}\right)$ functions such that $\left\|g_{N}\right\|_{2} \leqslant 1$, $\forall N \in \mathbb{N}^{+}\left(\|\cdot\|_{2}\right.$ meaning the $L_{\mu}^{2}\left(\mathbb{T}^{2}\right)$-norm $)$.

Using the quantities $K_{N, n}(\boldsymbol{x}, \boldsymbol{y})$ of Definition 4.2 we have that, for any given $A$ and $B$ measurable subsets of $\mathbb{T}^{2}$, and $N$ large enough, it holds

$$
\begin{aligned}
R_{N} & :=\left.\left|\int_{B} \mu(\mathrm{d} \boldsymbol{x}) g_{N}(\boldsymbol{x}) \mathcal{N} \int_{A} \mu(\mathrm{d} \boldsymbol{y})\right| K_{N, 1}(\boldsymbol{x}, \boldsymbol{y})\right|^{2}-\int_{B \cap T^{-1}(A)} \mu(\mathrm{d} \boldsymbol{x}) g_{N}(\boldsymbol{x}) \mid \\
& \leqslant \varepsilon_{B}(N) \quad,
\end{aligned}
$$

where $\varepsilon_{B}(N) \longrightarrow 0$ with $N \longrightarrow \infty$.

The symbol $\varepsilon_{B}$ does not imply any dependence of the bounding term $\varepsilon_{B}$ on the subset $B$; it is just a way of writing that will be of use in the following. 


\section{Proof of Lemma 5.1 :}

Resorting to the use of the characteristic functions $\mathcal{X}_{A}$ and $\mathcal{X}_{B}$, using triangular inequality and collecting terms, $R_{N}$ can be rewritten as

$$
\begin{aligned}
R_{N} & \leqslant\left.\int_{\mathbb{T}^{2}} \mu(\mathrm{d} \boldsymbol{x})\left|\mathcal{X}_{B}(\boldsymbol{x}) g_{N}(\boldsymbol{x})\right| \cdot\left|\mathcal{N} \int_{\mathbb{T}^{2}} \mu(\mathrm{d} \boldsymbol{y}) \mathcal{X}_{A}(\boldsymbol{y})\right| K_{N, 1}(\boldsymbol{x}, \boldsymbol{y})\right|^{2}-\mathcal{X}_{T^{-1}(A)}(\boldsymbol{x}) \mid \\
& =\left\|\mathcal{X}_{B} g_{N}\left[\mathcal{N} \int_{\mathbb{T}^{2}} \mu(\mathrm{d} \boldsymbol{y}) \mathcal{X}_{A}(\boldsymbol{y})\left|K_{N, 1}(\cdot, \boldsymbol{y})\right|^{2}-\mathcal{X}_{A}(T(\cdot))\right]\right\|_{1},
\end{aligned}
$$

and using the Cauchy-Schwartz inequality

$$
\leqslant\left\|\mathcal{X}_{B} g_{N}\right\|_{2} \cdot\left\|\mathcal{N} \int_{\mathbb{T}^{2}} \mu(\mathrm{d} \boldsymbol{y}) \mathcal{X}_{A}(\boldsymbol{y})\left|K_{N, 1}(\cdot, \boldsymbol{y})\right|^{2}-\mathcal{X}_{A}(T(\cdot))\right\|_{2} .
$$

Now we use the hypothesis, so that

$$
\left\|\mathcal{X}_{B} g_{N}\right\|_{2}^{2}=\int_{B}\left|g_{N}(\boldsymbol{x})\right|^{2} \mu(\mathrm{d} \boldsymbol{y}) \leqslant\left\|g_{N}\right\|_{2}^{2} \leqslant 1
$$

Putting together (53) and (54), and using Proposition 4.1 (with $f=\mathcal{X}_{A}$ and $n=1$ ) we get the result.

We are now in position to conclude with:

\section{Proof of Theorem 3 :}

Let us start to compute the expectation $\mathcal{P}_{\boldsymbol{i}}^{\mathrm{CS}}$. In terms of the quantity introduced in points (1-4) of the statement, equation (48) can be rewritten as

$$
\begin{aligned}
\mathcal{P}_{\boldsymbol{i}}^{\mathrm{CS}} & =\mathcal{N}^{n-1} \int_{E_{i_{0}}} \int_{E_{i_{1}}} \cdots \int_{E_{i_{n-1}}}\left\langle C_{\mathcal{N}}\left(\boldsymbol{x}_{0}\right)\left|\mathbb{1}_{\mathcal{N}}\right| C_{\mathcal{N}}\left(\boldsymbol{x}_{0}\right)\right\rangle \times \\
& \times \prod_{j=1}^{n-1}\left[\left|\left\langle C_{\mathcal{N}}\left(\boldsymbol{x}_{j}\right)\left|W_{T, N}\right| C_{\mathcal{N}}\left(\boldsymbol{x}_{j-1}\right)\right\rangle\right|^{2}\right] \mu\left(\mathrm{d} \boldsymbol{x}_{0}\right) \mu\left(\mathrm{d} \boldsymbol{x}_{1}\right) \cdots \mu\left(\mathrm{d} \boldsymbol{x}_{n-1}\right)
\end{aligned}
$$

and using normalization property for the state $\left|C_{\mathcal{N}}\left(\boldsymbol{x}_{0}\right)\right\rangle$ and resorting to Definition 4.2

$$
=\int_{E_{i_{n-1}}} \cdots \int_{E_{i_{1}}} \int_{E_{i_{0}}} \mu\left(\mathrm{d} \boldsymbol{x}_{n-1}\right) \times \prod_{j=1}^{n-1}\left[\mathcal{N}\left|K_{N, 1}\left(\boldsymbol{x}_{j}, \boldsymbol{x}_{j-1}\right)\right|^{2} \mu\left(\mathrm{d} \boldsymbol{x}_{j-1}\right)\right] .
$$

Now it start an iterate procedures, consisting of two points. 
1) consider the function

$$
g_{N}\left(\boldsymbol{x}_{1}\right):=\int_{E_{i_{n-1}}} \cdots \int_{E_{i_{3}}} \int_{E_{i_{2}}} \prod_{j=2}^{n-1}\left[\mathcal{N}\left|K_{N, 1}\left(\boldsymbol{x}_{j}, \boldsymbol{x}_{j-1}\right)\right|^{2} \mu\left(\mathrm{d} \boldsymbol{x}_{j}\right)\right] \quad:
$$

all the factors inside the integrals of (56) are positive, so that extending the integration domain and expliciting the form of $K_{N, 1}\left(\boldsymbol{x}_{j}, \boldsymbol{x}_{j-1}\right)$, we get the bound

$$
g_{N}\left(\boldsymbol{x}_{1}\right) \leqslant \int_{\mathbb{T}^{2}} \cdots \int_{\mathbb{T}^{2}} \int_{\mathbb{T}^{2}} \prod_{j=2}^{n-1}\left[\mathcal{N}\left|\left\langle C_{\mathcal{N}}\left(\boldsymbol{x}_{j}\right), W_{T, N} C_{\mathcal{N}}\left(\boldsymbol{x}_{j-1}\right)\right\rangle\right|^{2} \mu\left(\mathrm{d} \boldsymbol{x}_{j}\right)\right]=1
$$

from completeness and normalization, so that it follows $\left\|g_{N}\right\|_{2} \leqslant 1$.

2) By means of (56), equation (55) can be rewritten as

$$
\mathcal{P}_{\boldsymbol{i}}^{\mathrm{CS}}=\int_{E_{i_{1}}} \mu\left(\mathrm{d} \boldsymbol{x}_{1}\right) g_{N}\left(\boldsymbol{x}_{1}\right) \mathcal{N} \int_{E_{i_{0}}} \mu\left(\mathrm{d} \boldsymbol{x}_{0}\right)\left|K_{N, 1}\left(\boldsymbol{x}_{1}, \boldsymbol{x}_{0}\right)\right|^{2} .
$$

Now Lemma 5.1 guarantees that there exists a positive sequence $\varepsilon_{E_{i_{1}}}(N)$ such that,

$$
\left|\mathcal{P}_{\boldsymbol{i}}^{\mathrm{CS}}-\int_{E_{i_{1}} \cap T^{-1}\left(E_{i_{0}}\right)} \mu\left(\mathrm{d} \boldsymbol{x}_{1}\right) g_{N}\left(\boldsymbol{x}_{1}\right)\right| \leqslant \varepsilon_{E_{i_{1}}}(N)
$$

with $\varepsilon_{E_{i_{1}}}(N) \longrightarrow 0$ for $N \longrightarrow \infty$. By iterating $(n-1)$ )-times this procedure (consisting in isolating a single $K_{N, 1}\left(\boldsymbol{x}_{j}, \boldsymbol{x}_{j-1}\right)$ and grouping all the others in a single bounded function $\left.g_{N}\left(\boldsymbol{x}_{j}\right)\right)$ and using the triangle inequality for $|\cdot|$, we finally arrive to the result:

$$
\left|\mathcal{P}_{\boldsymbol{i}}^{\mathrm{CS}}-\mu\left(E_{i_{n-1}} \cap T^{-1}\left(E_{i_{n-2}}\right) \cap \cdots \cap T^{1-n}\left(E_{i_{0}}\right)\right)\right|=\left|\mathcal{P}_{\boldsymbol{i}}^{\mathrm{CS}}-\mu_{\hat{\boldsymbol{\imath}}}\right| \leqslant \varepsilon(N) \quad,
$$

with

$$
\varepsilon(N):=\sum_{\ell=1}^{n-1} \varepsilon_{E_{i_{\ell}}}(N) \longrightarrow 0 \quad \text { for } \quad N \longrightarrow \infty
$$

$\mu_{\boldsymbol{j}}$ meaning the classical probability of Section 5.1 and $\hat{\boldsymbol{\imath}}$ denoting the string $\boldsymbol{i}$ reversed, as in Definition 5.1.2.

We now define two density matrices, with the aim to compute their Von Neumann Entropy (see Section 5.2), that are both diagonal in the basis $\{|\boldsymbol{i}\rangle\}_{\boldsymbol{i} \in \Omega_{D}^{n}}$ of the $D^{n}$ di- 
mensional Hilbert space $\mathcal{H}_{D^{n}}$ :

$$
\rho:=\sum_{\boldsymbol{i} \in \Omega_{D}^{n}} \mu_{\hat{\boldsymbol{\imath}}}|\boldsymbol{i}\rangle\left\langle\boldsymbol{i}\left|\quad, \quad \sigma:=\sum_{\boldsymbol{i} \in \Omega_{D}^{n}} \mathcal{P}_{\boldsymbol{i}}^{\mathrm{CS}}\right| \boldsymbol{i}\right\rangle\langle\boldsymbol{i}|
$$

Resorting to the trace norm $\|A\|_{1}:=\operatorname{Tr}|A|=\operatorname{Tr} \sqrt{A^{\dagger} A}$, we use (57) to estimate $\|\rho-\sigma\|_{1}$, that is

$$
\Delta(n):=\|\rho-\sigma\|_{1} \leqslant D^{n} \varepsilon(N)
$$

Finally, by the continuity of the von Neumann entropy [29], we get

$$
|H(\rho)-H(\sigma)| \leqslant \Delta(n) \log D^{n}+\eta(\Delta(n))
$$

that is $\left|S\left(W_{T, N}, \mathcal{I}, \mathcal{E}, \rho, n\right)-S_{\mu}\left(\mathcal{E}_{[0, n-1]}\right)\right| \leqslant \Delta(n) \log D^{n}+\eta(\Delta(n))$, indeed the two Von Neumann entropy $H(\rho)$ and $H(\sigma)$ are nothing but the Shannon entropy of the refinements $\mathcal{E}_{[0, n-1]}$ of the classical partition (see Section 5.1), respectively the Shannon entropy (49) leading to the CS quantum entropy.

Since, from $n \leq \alpha \log N, D^{n} \leqslant N^{\alpha \log D}$, if we want the bound $D^{n} \varepsilon(N)$ to converge to zero with $N \longrightarrow \infty$, the parameter $\alpha$ has to be chosen accordingly.

By means of Theorem 3, a positive CS-entropy production is then associated to discrete systems whose continuous limit exhibit a positive KS-entropy production, which correspond in turn to the sum of all positive Lyapunov exponent of the continuous classical system, as stated by the Pesin's Theorem [15].

This positive CS-entropy production is entirely due to the dynamical component $H_{\text {dyn }}\left(W_{T, N}, \mathcal{I}, \mathcal{E}, \rho\right)$ of (52), being the measurement CS-entropy (51) equal to zero, as stated in the next proposition:

\section{Proposition 5.1}

Let $\mathcal{I}$ and $\mathcal{E}$ be the instrument, respectively the finite measurable partition of the statement of Theorem 3 and let $\rho$ be the tracial state $\frac{1}{\mathcal{N}} \mathbb{1}_{\mathcal{N}}$. There exists an $\alpha^{\prime}$ such that:

$$
\lim _{\substack{n, N \rightarrow \infty \\ n<\alpha^{\prime} \log N}} \frac{1}{n} S\left(\mathbb{1}_{\mathcal{N}}, \mathcal{I}, \mathcal{E}, \rho, n\right)=0
$$

\section{Proof:}

Performing a proof completely analogous to the one for Theorem 3, we find an $\alpha^{\prime}$ such 
that

$$
\lim _{\substack{n, N \rightarrow \infty \\ n<\alpha^{\prime} \log N}} \frac{1}{n}\left|S\left(\mathbb{1}_{\mathcal{N}}, \mathcal{I}, \mathcal{E}, \rho, n\right)-S_{\mu}\left(\mathcal{E}_{[0, n-1]}^{\prime}\right)\right|=0
$$

with $\mathcal{E}_{[0, n-1]}^{\prime}$ now given by $\mathcal{E}_{[0, n-1]}^{\prime}:=\bigvee_{j=0}^{n-1} \mathbb{1}^{j}(\mathcal{E})=\mathcal{E} \bigvee \mathcal{E} \bigvee \cdots \bigvee \mathcal{E}$ (see Section 5.1), so that

$$
S_{\mu}\left(\mathcal{E}_{[0, n-1]}^{\prime}\right)=S_{\mu}(\mathcal{E}) \leqslant \log D
$$

independent of $n$.

Now we use triangular inequality together with (59), obtaining

$$
\frac{1}{n} S\left(\mathbb{1}_{\mathcal{N}}, \mathcal{I}, \mathcal{E}, \rho, n\right) \leqslant \frac{1}{n}\left|S\left(\mathbb{1}_{\mathcal{N}}, \mathcal{I}, \mathcal{E}, \rho, n\right)-S_{\mu}\left(\mathcal{E}_{[0, n-1]}^{\prime}\right)\right|+\frac{\log D}{n}
$$

and so the result follows from (58).

\section{Conclusions}

In this work we studied the footprints of chaos present in classical dynamical systems on the two dimensional torus after a discretization has forced these systems to move on a regular lattice of spacing $\frac{1}{N}$, with finite number of sites $N^{2}$.

Discretizing is similar to quantizing; in particular, as for the classical limit $\hbar \rightarrow 0$, we have set up a solid theoretical framework to discuss the continuous limit $N \rightarrow \infty$.

Inspired by the semi-classical analysis, we developed an algebraic discretization technique by mimicking the well known Anti-Wick schemes of quantization, in particular we made use of a family of suitably defined Lattice States with properties that, in a quantum setting, are typical of Coherent States.

The result is the appearance of a logarithmic time-scale when the discrete hyperbolic systems tend to their continuous limit; namely, the continuous and discrete dynamics agree up to a breaking time which is proportional to the logarithm of the lattice spacing.

We also used the entropy production as a parameter of chaotic behaviour. In particular the notion of CS-quantum entropy has been used: this reproduce the classical metric entropy of Kolmogorov and Sinai if applied to classical continuous systems.

The CS-quantum entropy do converge to the KS invariant, but on logarithmic time scales too. 


\section{Acknowledgments}

The author wishes to thank Dr. F. Benatti for stimulating discussions and useful advice.

\section{A. Sketch of the proofs of Propositions 3.1, 3.2 and 3.3}

\section{Proof of Proposition 3.1 :}

1) - Let us start by considering matrices with positive trace, that is positive eigenvalues $\left(\lambda, \lambda^{-1}\right)$; the case of negative trace will be considered in next point (2). In the (nonorthogonal) reference system $\left(\hat{\boldsymbol{c}}_{1}, \hat{\boldsymbol{c}}_{2}\right)$ oriented along eigenvectors $\left(\left|\boldsymbol{e}_{+}\right\rangle,\left|\boldsymbol{e}_{-}\right\rangle\right)$, the timeevolution is described by

$$
\left(c_{1}, c_{2}\right) \underset{n \in \mathbb{N}}{\stackrel{T^{ \pm n}}{\longrightarrow}}\left(\lambda^{ \pm n} c_{1}, \lambda^{\mp n} c_{2}\right)
$$

thus orbits are simply given by $c_{1} c_{2}=$ Const., that in the reference system $(\hat{\boldsymbol{x}}, \hat{\boldsymbol{y}})$ reads as (11), indeed the relation between coordinates in the two systems is:

$$
\left(\begin{array}{l}
x \\
y
\end{array}\right)=\left(\begin{array}{ll}
1 & \cos \beta \\
0 & \sin \beta
\end{array}\right)\left(\begin{array}{l}
c_{1} \\
c_{2}
\end{array}\right) \text {. }
$$

Among these orbits, we choose the two that are tangent (and so closest) to the unit ball $B_{T}(0)$ : of course they remain tangent and closest even during evolution $B_{T}(0) \longmapsto B_{T}(n)$ and so they give us the the right expression for the surrounding orbits of $B_{T}^{(n)}$, that is (13).

By means of (61) and (62) we have an expression for the $\pm n$-evolved unit ball, that is $B_{T}(n)$; among its surface's points we choose the farthest ones and we determine their norm, getting the expression for $D_{T}(n)$ contained in (14).

Now we use the expression $\sinh ^{-1}(q)=\log \left(\sqrt{q^{2}+1}+q\right)$, that holds for all $q>0$, in particular for $q=\left(\lambda^{n}-\lambda^{-n}\right) / \sin \beta(\sin \beta>0)$, so that from (14) we get for $D_{T}(n)$ the expression given by (15), that shows the monotonicity in $n$ of this function; this monotonicity, together with the definitions $(10)$ of $B_{T}^{(n)}$, give us the equivalence between $D_{T}^{(n)}$ and $D_{T}(n)$.

The linear matrix action $T$ map the unit ball $B_{T}(0)$ in the ellipse $B_{T}(1)$ an $D_{T}(1)$ is its major semi-axis; from Definition 3.2, we have

$$
\eta^{2}=\sup _{|\boldsymbol{v}\rangle \in \mathbb{R}^{2}}\left\langle\boldsymbol{v}\left|T^{\dagger} T\right| \boldsymbol{v}\right\rangle=\sup _{|\boldsymbol{v}\rangle \in \mathbb{R}^{2}} \| T|\boldsymbol{v}\rangle \|_{\mathbb{R}^{2}}^{2}=\left[D_{T}(1)\right]^{2}
$$

so that $\eta=D_{T}(1)$ and (12) follows from expression (14), with $n=1$. 
Expressions in (16) can be easily deduced from (14).

2) - Let us now notice that every map $T$, whose trace is negative, may be written as the composition of $-\mathbb{1}_{2}$ (the identity map) with the map $-T$, which has positive trace;

the same holds true for the iterates $\left\{T^{k}\right\}_{k}$ odd . Since multiplying by $-\mathbb{1}_{2}$ amounts to perform the transformation $(x, y) \longmapsto(-x,-y)$, both the orbits (11) and the surrounding surface (12), which exhibit a central symmetry, remain the same also for negative trace maps. The same argument can be applied to the diameter $D_{T}(n)$ of $(14)$, which are invariant for coordinates reflection too.

\section{Proof of Proposition 3.2 :}

Let us consider matrices $T$ with $\operatorname{Tr} T=2$, that is $t=1$, being the case $t=-1$ equivalent, as it is possible to prove in the same way of point (2) of the proof of Proposition 3.1. In the orthogonal reference system $(\hat{\boldsymbol{x}}, \hat{\boldsymbol{y}})$ of the statement, the action of $T^{n}$ is described by a matrix in Jordan canonical form, that is

$$
\left(\begin{array}{l}
x \\
y
\end{array}\right) \underset{T^{n}}{\longrightarrow}\left(\begin{array}{l}
x^{\prime} \\
y^{\prime}
\end{array}\right)=\left(\begin{array}{cc}
1 & n J^{\prime} \\
0 & 1
\end{array}\right)\left(\begin{array}{l}
x \\
y
\end{array}\right),
$$

where $J^{\prime}=t_{12}-t_{21}$, thus orbits are simply given by $y=$ Const. In order to apply the argument of point (2) of proof of Proposition 3.1, when $t=-1$, we endow this class of orbits with a coordinate reflection symmetry, and this leads to equation (17).

Among these orbits, we choose the one that is tangent (and so closest) to the unit ball $B_{T}(0)$ : of course it remains tangent an closest even during evolution $B_{T}(0) \longmapsto B_{T}(n)$ and so it give us the the right expression for the surrounding orbit of $B_{T}^{(n)}$, that is (18).

By means of (63) we have an expression for the $\pm n$-evolved unit ball, that is $B_{T}(n)$; among its surface's points we choose the farthest ones and we determine their norm, getting the expression for $D_{T}(n)$ contained in $(20)$, with $J=\left|J^{\prime}\right|$.

Using once more the expression $\sinh ^{-1}(q)=\log \left(\sqrt{q^{2}+1}+q\right)$, that holds for all $q>0$, in particular for $q=n J$, from (20) we get for $D_{T}(n)$ the expression given by (21); using monotonicity we get the equivalence $D_{T}^{(n)}=D_{T}(n)$.

From $\eta=D_{T}(1)$ (see proof of Proposition 3.1), equation (19) can be earned from expression (20), with $n=1$.

Expressions in (22) and (23) can be easily deduced and verified from (20).

\section{Proof of Proposition 3.3 :}

The semi-trace $t$ of the matrix $T$ can only assume values in $\left\{-\frac{1}{2}, 0, \frac{1}{2}\right\}$, indeed all entries 
of $T$ are integer and $|t|<1$. We read from equation (8) that $t=\cos \phi$ and so we have for $\phi$ the only possible values $\left\{ \pm \frac{2}{3} \pi, \pm \frac{1}{2} \pi, \pm \frac{1}{3} \pi\right\}$; everyone of these values make the timeevolution periodic, as it can be deduced from equation (8). All these cases are similar; we now prove the statement for $t=\frac{1}{2}$.

$\boldsymbol{t}=\frac{1}{2}$ - We have $\phi= \pm \frac{1}{3} \pi$ and so we get from equation (8) that $T^{3}=-\mathbb{1}_{2}$. The period of evolution is six and the sequence of $T$-power is equivalent to $\mathbb{1}_{2}, T,-T^{-1},-\mathbb{1}_{2}$, $-T, T^{-1}, \mathbb{1}_{2}$ and so on.

By using equation (9) of Definition 3.2 we see that the sequence $\left\{B_{T}(n)\right\}_{n \in \mathbb{N}}$ of $n$-evolved ball is equivalent to $B_{T}(0), B_{T}(1), B_{T}(-1), B_{T}(0), B_{T}(1), B_{T}(-1) \ldots$, thus, the sequence of diameter $\left\{D_{T}(n)\right\}_{n \in \mathbb{N}}$, is given by $D_{T}(0), D_{T}(1), D_{T}(-1) \ldots$

As argued in the proof of Proposition 3.1 (point 1), $D_{T}(1)=\eta$; moreover $D_{T}(-1)=$ $\eta$ too. Indeed, as the spectra of $|T|$ consists of the two eigenvalue $\left(\eta, \eta^{-1}\right)$, the same is true for the spectra of $\left|T^{-1}\right|$.

Using the last observation, the sequence of diameter becomes $0, \eta, \eta, 0, \eta, \eta \ldots$ and so equations (24-25) hold true for the case $t=\frac{1}{2}$.

The cases $\boldsymbol{t}=-\frac{\mathbf{1}}{\mathbf{2}}$ and $\boldsymbol{t}=\mathbf{0}$ can be proved in a similar way.

\section{References}

[1] G.M. Zaslavsky, Chaos in Dynamic Systems, Harwood Academic Publ., Chur, 1985.

[2] M.--J. Giannoni, A. Voros and J. Zinn-Justin, editors, Chaos and Quantum Physics, volume 1989 Les Houches Session LII of Les Houches Summer School of Theoretical Physics, Amsterdam, London, New York, Tokyo, 1991, North-Holland.

[3] R. Devaney, An Introduction to Chaotic Dynamical Systems, Addison-Wesley, Reading, MA, 1989.

[4] S. Wiggins, Dynamical Systems and Chaos, Springer-Verlag, New York, 1990.

[5] H.G. Schuster, Deterministic Chaos, VCH, Weinheim, 3rd edition, 1995.

[6] G. Casati and B. Chirikov, Quantum Chaos. Between Order and Disorder, Cambridge University Press, Cambridge, 1995.

[7] A. Katok and B. Hasselblatt, Introduction to the Modern Theory of Dynamical Systems, Encyclopedia of Mathematics and its Applications, Cambridge University Press, Cambridge, 1999.

[8] J. M. Bouclet and S. De Bievre, Long time propagation and control on scarring for perturbed quantized hyperbolic toral automorphisms, Preprint math-ph/0409069, 2004. 
[9] A. Fannjiang, S. Nonnenmacher and L. Wolowski, Dissipation time of quantized toral maps, Preprint math-ph/0406055, 2004.

[10] R. Schubert, Semiclassical behaviour of expectation values in time evolved lagrangian states for large times, Preprint math.MP/0402038, 2004.

[11] V. Cappellini, Quantum Dynamical Entropies and Complexity in Dynamical Systems, PhD thesis, University of Trieste, 2004, Preprint math-ph/0403035.

[12] F. Bonechi and S. De Bièvre, Controlling strong scarring for quantized ergodic toral automorphisms, Duke Math. J. 3, 571-587 (2003).

[13] R.F. Werner, The classical limit of quantum theory, Preprint quant-ph/9504016, 1995.

[14] F. Benatti, V. Cappellini, M. De Cock, M. Fannes and D. Vanpeteghem, Classical limit of quantum dynamical entropies, Rev. Math. Phys. 15(8), 847-875 (2003).

[15] R. Mañé, Ergodic Theory and Differentiable Dynamics, Springer-Verlag, Berlin, 1987.

[16] V. M. Alekseev and M. V. Yakobson, Symbolic dynamics and hyperbolic dynamical systems, Phys. Rep. 75, 287-325 (1981).

[17] A. Connes, H. Narnhofer and W. Thirring, Dynamic entropy of $\mathrm{C}^{*}$-algebras and Von Neumann algebras, Comm. Math. Phys. 112, 691-719 (1987).

[18] R. Alicki and M. Fannes, Defining quantum dynamical entropy, Lett. Math. Phys. 32, 75-82 (1994).

[19] W. Słomczyński and K. Życzkowski, Quantum chaos: an entropy approach, J. Math. Phys. 35(11), 5674-5700 (1994).

[20] F. Benatti, V. Cappellini and F. Zertuche, Quantum dynamical entropies in discrete classical chaos, J. Phys. A: Math. Gen. 37(1), 105-130 (2004).

[21] F. Benatti and V. Cappellini, Continuous limit of discrete Sawtooth Maps and its algebraic framework, J. Math. Phys. 46(6), 062702 (2005).

[22] O. Bratteli and D. W. Robinson, Operator algebras and quantum statistical mechanics (v.1: $C^{*}$ - and $W^{*}$-algebras, symmetry groups, decomposition of states), Texts and monographs in physics, Springer-Verlag, New York, 1979.

[23] E. Hewitt and K. Stromberg, Real and Abstract Analysis, Springer-Verlag, Berlin, 2nd edition, 1969.

[24] W. Rudin, Real and Complex Analysis, McGraw-Hill, New York, 3rd edition, 1987.

[25] J. Marklof and Z. Rudnick, Quantum unique ergodicity for parabolic maps, GAFA, Geom. Funct. Anal. 10, 1554-1578 (2000). 
[26] F Riesz and B. Sz.-Nagy, Functional Analysis, Frederick Ungar Publishing Co., New York, 1955.

[27] J. Ford and M. Ilg, Eigenfunctions, eigenvalues, and time evolution of finite, bounded, undriven, quantum systems are not chaotic, Phys. Rev. A 45(9), 61656173 (1992).

[28] H. Narnhofer, Quantized Arnold cat maps can be entropic K-systems, J. Math. Phys. 33, 1502-1510 (1992).

[29] M. Fannes, A continuity property of the entropy density for spin lattice systems, Comm. Math. Phys. 31, 291-294 (1973). 